\title{
Echinoderms from Santa Catarina, southern Brazil: an update on biodiversity and distribution
}

\author{
Nataly Nunes Slivak'; Alberto Lindner ${ }^{2} \&$ Helena Picolli Romanowski ${ }^{3}$
}

\begin{abstract}
1 Universidade Federal do Rio de Janeiro (UFRJ), Museu Nacional (MN), Programa de Pós-Graduação em Zoologia. Rio de Janeiro, RJ, Brasil. ORCID: https://orcid.org/0000-0001-6109-5697. E-mail: nataly.slivak@gmail.com (corresponding author)

2 Universidade Federal de Santa Catarina (UFSC), Departamento de Ecologia e Zoologia (ECZ). Florianópolis, SC, Brasil. ORCID: https://orcid.org/0000-0003-3288-0885. E-mail: alberto.lindner@ufsc.br

${ }^{3}$ Universidade Federal do Rio Grande do Sul (UFRGS), Instituto de Biociências (IBI0), Departamento de Zoologia. Porto Alegre, RS, Brasil. ORCID: https://orcid.org/0000-0001-8342-4607.E-mail: hpromano@ufrgs.br
\end{abstract}

\begin{abstract}
We provide an update, based on literature records, on biodiversity and distribution of echinoderms along the state of Santa Catarina, Brazil. Sixty-one echinoderms taxa are reported, in 36 families. We present information on habitat distribution, taxonomy and possible threats to these species. The highest number of echinoderm records was between $25^{\circ} 19^{\prime} S$ and $28^{\circ} 11^{\prime} S$ latitudes, and sites of the Continental Shelf and the Reserva Biológica Marinha of Arvoredo area had the most species records ( 30 and 26 species, respectively). In addition, Santa Catarina is the southernmost record, in the Atlantic Ocean, for 40 echinoderm species; of these, 10 are on the regional list of endangered species. Eight species are endemic to Brazil and one ophiuroid species was reported herein for the first time at Santa Catarina: Ophiacantha pentacrinus Lütken, 1869.
\end{abstract}

Keywords. Echinodermata; Threatened species; Geographical distribution; Atlantic biodiversity; Faunistic survey.

\section{INTRODUCTION}

There are about 7,000 echinoderm species worldwide, of which 347 have been reported in Brazil (Ventura et al., 2006, 2013). The group is represented by sea stars (Asteroidea), sea urchins (Echinoidea), sea cucumbers (Holothuroidea), sea lilies and feather stars (Crinoidea), and brittle stars (Ophiuroidea). Echinoderms, such as starfishes and sea urchins, are important predators and herders that play key roles in the functioning of shallow-water marine ecosystems (Paine, 1966; Lessios, 1988; Birkeland, 1989). Over the years, echinoderms became targets of anthropic action. Sea urchins and sea cucumbers, for instance, are commercially popular in several regions for human consumption and supposed medicinal properties (Pangestuti \& Arifin, 2017). In Brazil, these organisms are also collected and sold as a decorative artifact, such as the starfish Oreaster reticulatus (Linnaeus, 1758) (Dias et al., 2011; Martins et al., 2012c; Alves et al., 2018), currently in vulnerable status in the endangered species book (ICMBio, 2018).

The spatial arrangement of continents and oceans, combined with the influence of temperature and latitudinal gradients, local circula- tion patterns, and the properties of water divide the oceans into a series of provinces or biogeographic regions with characteristic assemblies (Floeter \& Soares-Gomes, 1999). The Brazilian biogeographic province extends from the Amazon River to Santa Catarina in southern Brazil (Floeter et al., 2008; Briggs \& Bowen, 2012). The state of Santa Catarina, from Itapoá $\left(25^{\circ} 59^{\prime} \mathrm{S}\right)$ to Passo de Torres $\left(29^{\circ} 19^{\prime} \mathrm{S}\right)$, represents the southern limit for the distribution of many shallow-water marine species, such as the starfish Coscinasterias tenuispina (Lamarck, 1816) (ICMBio, 2016), the marine sponge Dragmaxia anomala Carvalho \& Hajdu, 2004 (Carraro, 2012), the mollusk Tambja stegosauriformis Pola, Cervera \& Gosliner, 2005 (Padula et al., 2011; Cunha et al., 2016), zooxanthellate scleractinian corals (e.g., Capel et al., 2012; Lindner et al., 2017) and numerous species of other invertebrate groups and tropical reef fish (e.g., Anderson et al., 2015).

The history of studies on echinoderms in Brazil goes back to the first report of echinoderms in 1648 (see Marcgrave, 1942; Ventura et al., 2013). The following records were those of Verrill (1868), Rathbun (1879), Ludwig (1882), and those from the southern coast of Brazil by Müller (1898) (Tiago, 1998; Hadel et al., 1999; Tiago \& Ditadi, 
2001; Ventura et al., 2006), who recorded the crinoid Antedon carinatus (Lamarck, 1816) [currently Tropiometra carinata (Lamarck, 1816)] and the sand-dollar Encope emarginata (Leske, 1778) in the state of Santa Catarina.

In the twentieth century, researcher Luiz Roberto Tommasi, from Instituto Oceanográfico da Universidade de São Paulo, published a range of studies on echinoderms. Tommasi contributed to the description and new records of several species off Santa Catarina (Tommasi, 1964, 1965, 1966, 1970a, b, 1971, 1999). A conference abstract (Oliveira et al., 1987; Oliveira, 1990) and an unpublished honor thesis on starfishes and ophiuroids (Oliveira, 1989) provided additional records of several other species of echinoderms for the state. Subsequently, Xavier (2010), based on a review of the literature, published the first checklist of echinoderms from Santa Catarina, with 34 species in 16 different sites. However, several species of echinoderms recorded by Oliveira et al. (1987) and Oliveira (1989) were not included (Xavier, 2010). More recently, additional records of echinoderms for Santa Catarina were reported by Labbé-Bellas et al. (2016) and Bueno et al. (2018). Based on these studies, herein we provide an updated checklist of echinoderms for Santa Catarina.

\section{MATERIAL AND METHODS}

\section{Study area}

The coast of the state of Santa Catarina $\left(25^{\circ} 59^{\prime}\right.$ to $29^{\circ} 19^{\prime} \mathrm{S}$ ) is 561.4 kilometers long, and comprises more than 500 beaches, 47 islands, mangroves, lagoons, bays and other ecosystems (Branco et al., 2004; Rodrigues et al., 2004; Anderson et al., 2015). The central-northern coast of the state is under the influence of upwelling of the South Atlantic Central Water (SACW) (Carvalho et al., 1998). It is a phenomenon of biological and ecological importance, being responsible for the increase of primary production, more pronounced in the spring and summer seasons (Gherardi et al., 2008).

\section{Data}

An extensive literature survey on echinoderm records was performed with literature records available on the search sites Google Scholar, Capes platform, Scientific Electronic Library Online (SciELO), bibliographic database of the Federal University of Rio Grande do Sul (LUME), Library System from Federal University of Paraná (Portal UFPR). In addition, the search included conference proceedings, scientific manuscripts, undergraduate thesis, master thesis, PhD thesis, books, and book chapters, endangered species lists and technical reports. Data from species collected in Santa Catarina coast also were extracted and listed from national and foreigner museums database, Global Biodiversity Information Facility (GBIF) platforms, Ocean Biogeographic Information System (OBIS) and the collaborative network of museums SpeciesLink.
The search criteria used was "localities in Santa Catarina", and the keywords used in online searches, "Echinodermata", "Echinoderms", "Echinoderms from Santa Catarina", "Marine fauna of Santa Catarina", "Diversity of fauna in Santa Catarina", "Equinodermos de Santa Catarina", "Fauna marinha de Santa Catarina", "South Atlantic echinoderms".

We also included the following information about species:

Threatened species: The species in this study were classified in three IUCN (International Union for Conservation of Nature) categories, NE (Not Evaluated), VU (Vulnerable), $\mathrm{CR}$ (Critically Endangered) and one criterion, A (population reduction (past, present and/or projected for the future) IUCN, 2020). The data were obtained from Livro Vermelho da Fauna Brasileira Ameaçada de Extinção (ICMBio, 2018), Atlas da Fauna Brasileira Ameaçada de Extinção em Unidades de Conservação Federais (Nascimento \& Campos, 2011) and Lista das Espécies da Fauna Ameaçada de Extinção em Santa Catarina (IMA, 2011).

Habitat distribution: Eight different habitat types are provided. The Soft bottom (SB) is the zone covered essentially by sandy and muddy sediments. Rocky bottom (RB) is the zone associated with the presence of rocky substrate such as rocky shores and isolated boulders. Biologic Bottom (BB) is composed essentially by sponge, coral, bryozoans, other echinoderms and polychaetes colonies. Calcareous Algae (CA). Seagrass bed (SeB). Shells bottom (ShB). Gravel bottom (GB). Not Informed (NI).

Geographic range: The data were obtained from GBIF (online database), WoRMS (online database), Oliveira et al. (2010), Alvarado \& Solís-Marín (2013) and Bueno et al. (2018). Abbreviations are as follow SWA = Southwestern Atlantic Ocean; SEA = Southeastern Atlantic Ocean; $\mathrm{GM}=$ Gulf of Mexico; CS = Caribbean Sea; CAA = Central American Atlantic Coast; NWA = Northwestern Atlantic Ocean; NEA = Northeastern Atlantic Ocean; $\mathrm{ARO}=$ Arctic Ocean; NS = North Sea; MS = Mediterranean Sea; $\mathrm{RS}=$ Red Sea; SWI = Southwestern Indic Ocean; SEI = Southeastern Indic Ocean; NWI = Northwestern Indic Ocean; NEI = Northeastern Indic Ocean; IPO = IndoPacific Ocean; NEP = Northeastern Pacific Ocean; NWP = Northwestern Pacific Ocean; CP = Central Pacific Ocean; SWP = Southwestern Pacific Ocean; SEP = Southeastern Pacific Ocean; CG = Circumglobal; $A O=$ Antarctic Ocean; DSt $=$ Drake Strait; WS = Weddell Sea; BR = Brazil. In addition, those species whose southern limit of geographic distribution, in the Atlantic Ocean, is Santa Catarina coast were marked.

Record type: The following criteria were used herein: LIT $=$ in litteris (record from the literature without illustration or photograph); $\mathrm{PHO}=$ Photographs (record from the literature with specimens illustrated by photographs); SIG = In situ observation by authors. The species reported in unpublished studies (Ph.D. thesis, M.Sc. thesis and undergraduate thesis) were only considered 
herein if those could be identified on the basis of photographs or illustrations provided.

Acronyms: Scientific institutions (Echinodermata Collection (EQMN) of the Museu Nacional da Universidade Federal do Rio de Janeiro (MN/UFRJ); Zoological Collection of the Museum of Comparative Zoology from Harvard University (MCZ-IZ); Zoological Invertebrates of the Muséum National D'Histoire Naturelle, Paris (MNHN-FR); Ophiuroidea Collection of the Museu de Zoologia da Universidade de Campinas (ZUEC-OPH); Collection of Invertebrate Zoology of the California Academy of Science (CAS-IZ); Invertebrate Collection of the Museu de Zoologia da Universidade de São Paulo (MZUSP); Collection of Invertebrates of the Smithsonian Institution National Museum of Natural History (ISNMNH-IZ); Collection of the Laboratório de Praias Arenosas (LABMAR) of the Universidade Federal do Paraná (UFPR); Universidade do Vale do Itajaí (UNIVALI); Instituto do Meio Ambiente de Santa Catarina (IMA); Instituto Chico Mendes de Conservação e Biodiversidade (ICMBio)) and Scientific databases online (World Marine of Register Species (WoRMS); Ocean Biogeographic Information System (OBIS); Global Biodiversity Information Facility (GBIF)).

\section{RESULTS}

The echinoderm species search resulted in 444 records for the state of Santa Catarina. One hundred and fifty one records are in scientific manuscripts, 90 in master thesis, $\mathrm{PhD}$ thesis and undergraduate thesis, 45 in conference proceedings, 41 in endangered species lists, 27 in book chapters and 16 in technical reports (see Appendix 1). The scientific databases OBIS, GBIF and SpeciesLink resulted in 60 records and 14 in zoological collections from foreigner institutions (see Appendix 2).

In total, the search revealed 66 echinoderms taxa: 61 species, three subspecies and two genera (without specific classification). All of them were classified in five classes and 36 families (Table 1).

The largest number of recorded specimens belongs to the Class Ophiuroidea (33 species), followed by Asteroidea (12 species and three subspecies) and Echinoidea (12 species and two genera), Holothuroidea (two species) and Crinoidea (two species).

One echinoderm species not previously reported in the literature for the coast of Santa Catarina is reported herein: Ophiacantha pentacrinus Lütken, 1869.

\section{Order Ophiacanthida, Family Ophiacanthidae}

Ophiacantha pentacrinus Lütken, 1869. Three individuals were sampled by Onboard Fleet Observers Program (Programa de Observadores de Bordo na Frota Arrendada - PROA) executed by the fishing study group (Grupo de Estudos Pesqueiros - GEP) from University of Vale do Itajaí (Universidade do Vale do Itajaí - UNIVALI).
The specimens were identified by Dr. Michella Borges, specialist and curator of the Echinoderms Collection from the Museum of Zoology of the University of Campinas (Museu de Zoologia da Universidade Estadual de Campinas - ZUEC). The specimens were labeled as ZUEC-OPH 1868; ZUEC-OPH 1871 and ZUEC-OPH 1872 (see Appendix 2). The previous southernmost record was the state of Paraná (Borges et al., 2015), in this study we extend it to the Santa Catarina coast.

Seventy-seven percent of Echinoderms species recorded occur on sandy substrate (SB) (Table 1). Some ophiuroids species, such as Astrocyclus caecilian, were observed on gorgonians. Regular equinoids and starfish species, such as Narcissia tigonaria, prefer rocky bottoms. However, irregular equinoids, such as Clypeaster subdepressus and the sea cucumber Holothuria (Halodeima) grisea, occur frequently on gravel and sandy substrates (Fig. 1).

Sixteen echinoderm species listed are present in the endangered species list of Santa Catarina (IMA, 2011), and seven of them are in the Brazilian Red List (ICMBio, 2018).

The species were recorded in 135 different sites off Santa Catarina (Fig. 2) and the highest number of echinoderm records was between $25^{\circ} 19^{\prime} S$ and $28^{\circ} 11^{\prime} \mathrm{S}$ latitudes. Sites of the continental shelf and the Reserva Biológica Marinha of Arvoredo area had most of the records (30 and 26 species, respectively) (Tables 1 and 2). In addition, we observed that Santa Catarina is the southernmost record, in the Atlantic Ocean, for 40 echinoderm species listed herein (19 Ophiuroidea, 12 Echinoidea, five Asteroidea, two Crinoidea and two Holothuroidea) (Table 1). Eight species are endemic to the Brazilian coast: the ophiuroids Amphiura deichmanni, Amphiura muelleri, Ophiothrix brasiliensis, Ophiothrix rathbuni, Ophiothrix tommasii, Ophiothrix troscheli, Ophiomisidium tommasii, Ophiomastus satelitae.

\section{DISCUSSION}

In this study, we updated the list of echinoderms reported for Santa Catarina, including information on taxonomy, habitat and possible threats. In Brazil, echinoderm diversity comprised 347 species, $5 \%$ of the world total (about 7,000 species) (Ventura et al., 2013). For the state of Santa Catarina, Xavier (2010) published a checklist with 34 echinoderm species. Subsequently, Bueno et al. (2018) identified 42 echinoderms species at the northern part of Santa Catarina. In the present study, we assembled 67 species records (Table 1, Fig. 2), a result that reveals that the state of Santa Catarina comprises $19 \%$ of the total echinoderm diversity in Brazil. In São Paulo and Bahia, two other states for which checklists are available, $34.2 \%$ and $23.14 \%$ of the echinoderm diversity in Brazil is present, respectively (Hadel et al., 1999; Alves \& Cerqueira, 2000; Tiago \& Ditadi, 2001; Netto et al., 2005; Manso, 2004; Magalhães et al., 2005; Borges, 2006; Martins et al., 2012a, b, c; Miranda et al., 2012; Queiroz et al., 2013; Bueno et al., 2018). 


\begin{tabular}{|c|c|c|c|c|c|c|c|c|c|c|c|c|}
\hline 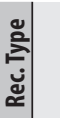 & $\begin{array}{l}\frac{0}{\pi} \\
\frac{0}{2} \\
\frac{0}{2}\end{array}$ & 辛: & 言 & 홀 & 产 & : & 亲 & 홍 & 홀 & 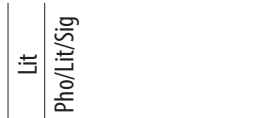 & $\frac{5}{\frac{5}{2}}$ & 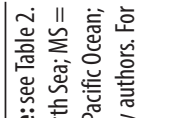 \\
\hline & 类 & 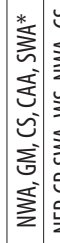 & 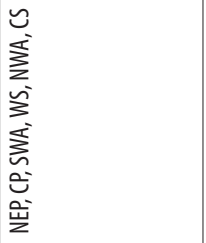 & 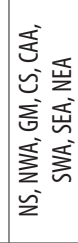 & 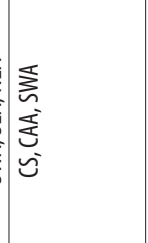 & 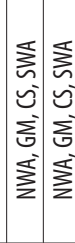 & 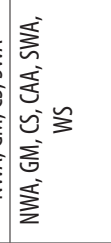 & 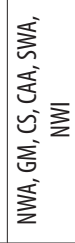 & 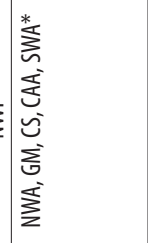 & 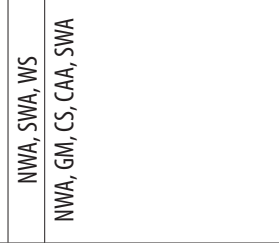 & 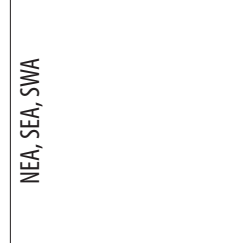 & 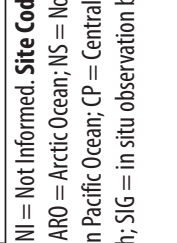 \\
\hline & 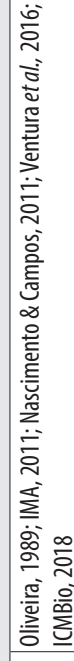 & 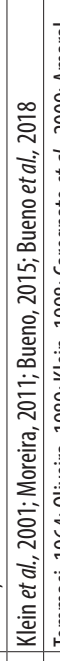 & 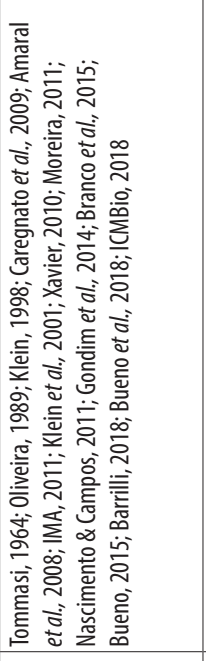 & 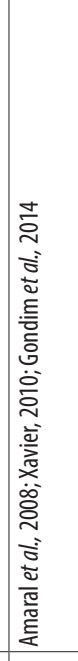 & 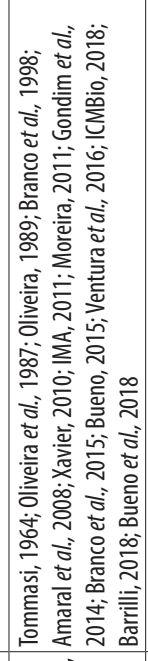 & 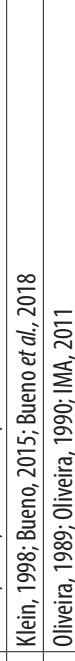 & 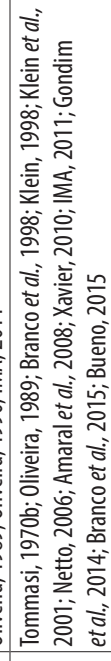 & 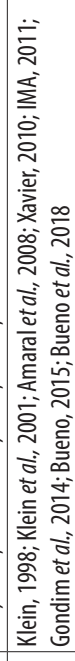 & 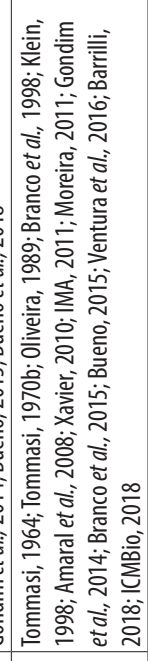 & 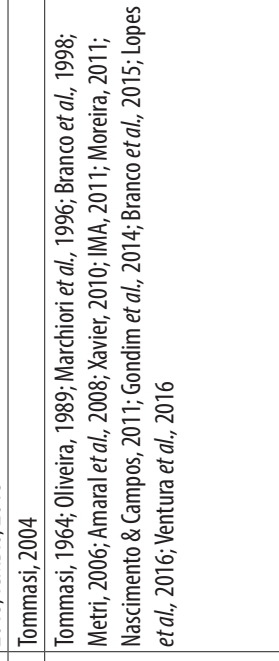 & 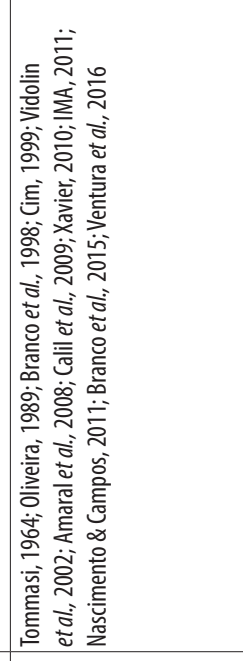 & 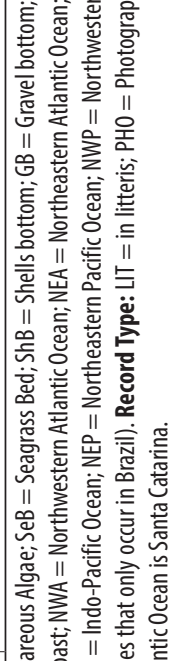 \\
\hline 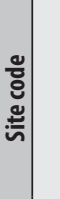 & 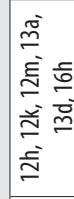 & $\frac{\pi}{2}$ & 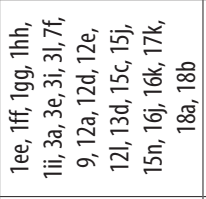 & & 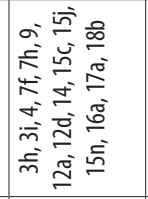 & : & 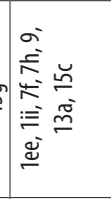 & 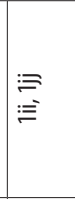 & 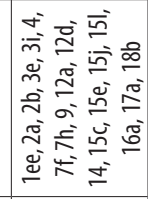 & 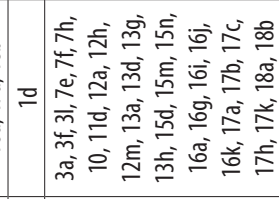 & 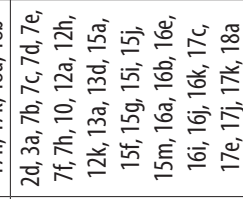 & 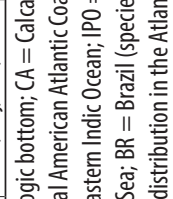 \\
\hline 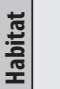 & $\begin{array}{l}\cong \\
\approx \\
\approx\end{array}$ & $\approx s$ & $\cong$ & 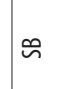 & 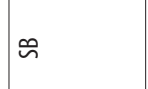 & $\cong \approx$ & $\begin{array}{l}\infty \\
\infty \\
\curvearrowleft\end{array}$ & $\cong$ & $\approx$ & 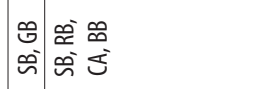 & $\begin{array}{l}\dddot{\varkappa} \\
\ddot{n}\end{array}$ & $l_{0}^{11} \sum^{2}$ \\
\hline$\underline{z}$ & 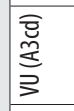 & $\mathrm{w}$ & $\frac{\overline{0}}{\frac{5}{5}}$ & 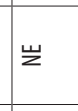 & $\frac{\bar{\Xi}}{\frac{3}{2}}$ & 岁 & u & 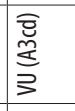 & 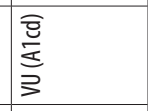 & 岁 & 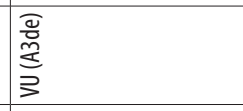 & 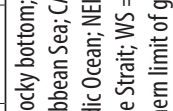 \\
\hline 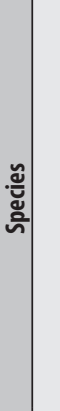 & 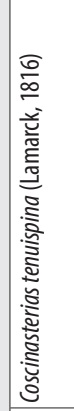 & 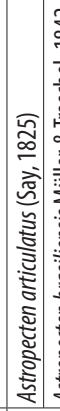 & 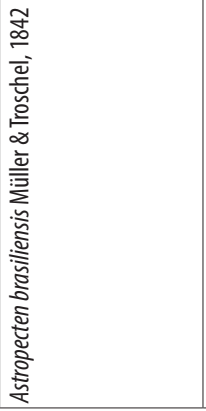 & 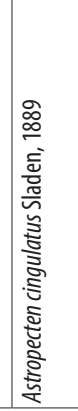 & 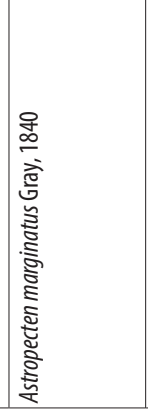 & 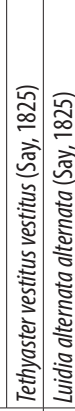 & 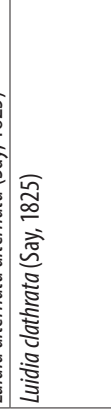 & 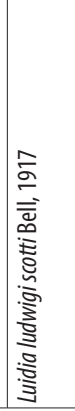 & 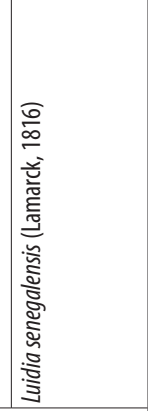 & 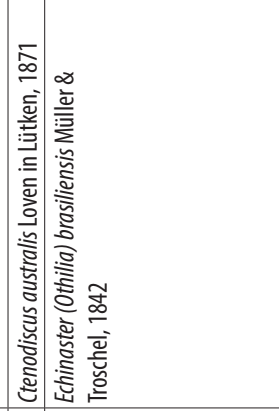 & 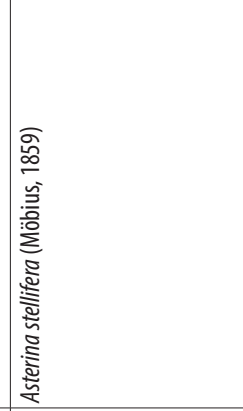 & 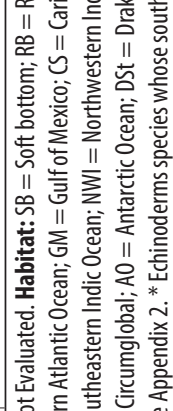 \\
\hline 츨 & 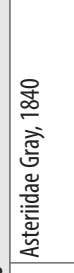 & 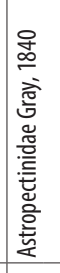 & & & & 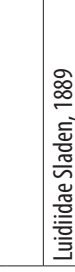 & & & & 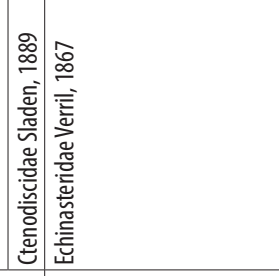 & 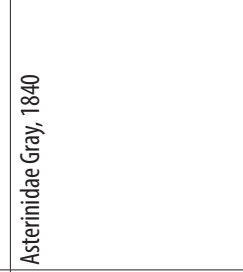 & 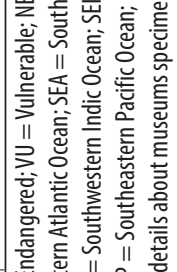 \\
\hline 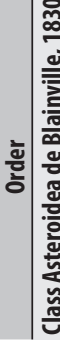 & 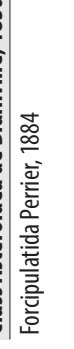 & 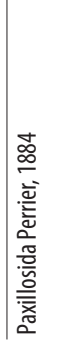 & & & & & & & & 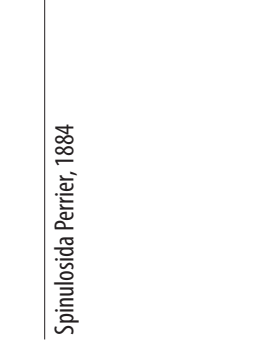 & 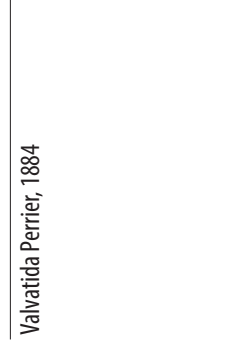 & 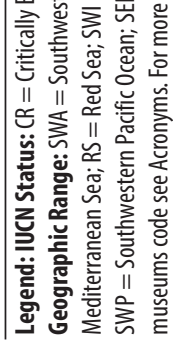 \\
\hline
\end{tabular}




\begin{tabular}{|c|c|c|c|c|c|c|c|c|c|c|c|c|c|c|}
\hline & $\frac{\sqrt{2}}{\frac{5}{2}}$ & 章 & & $\Xi$ & 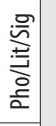 & 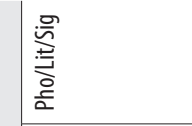 & 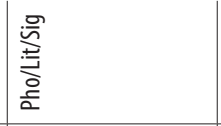 & 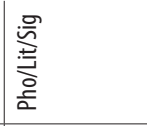 & \begin{tabular}{|l} 
올 \\
言 \\
음
\end{tabular} & 홀 & 䇏 & 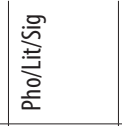 & 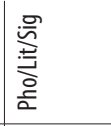 & 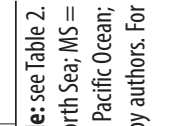 \\
\hline & 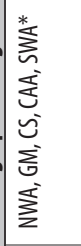 & 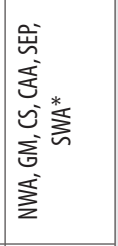 & & | & 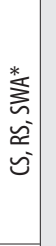 & 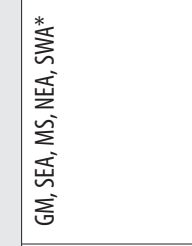 & 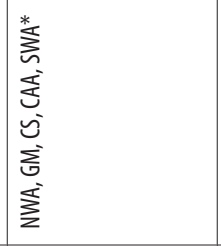 & 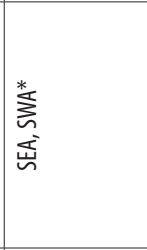 & 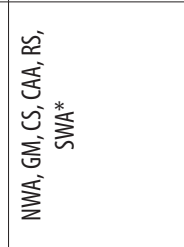 & 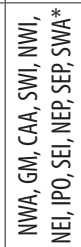 & 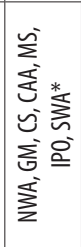 & 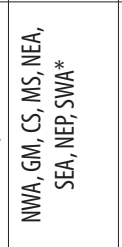 & 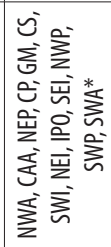 & 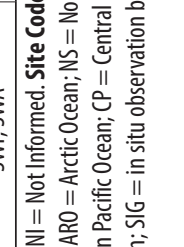 \\
\hline & 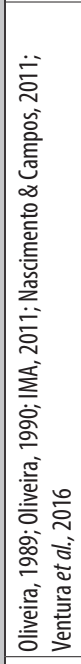 & 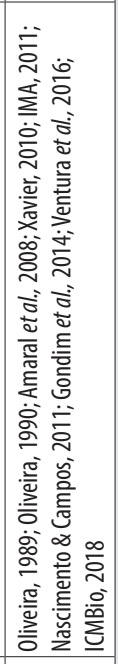 & & 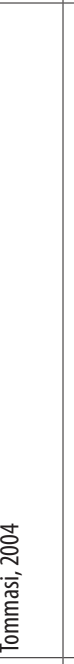 & 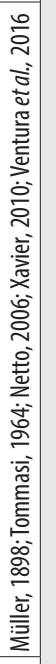 & 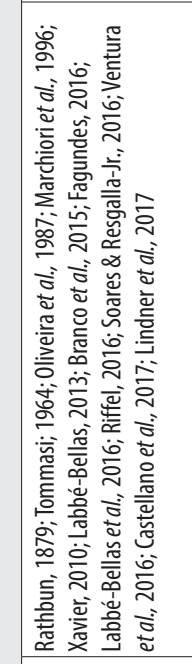 & 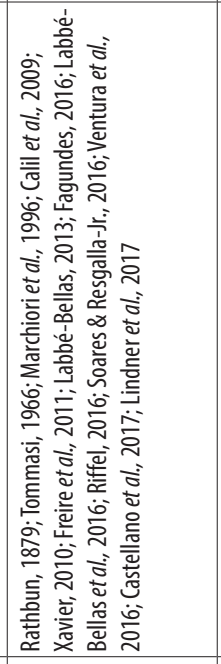 & 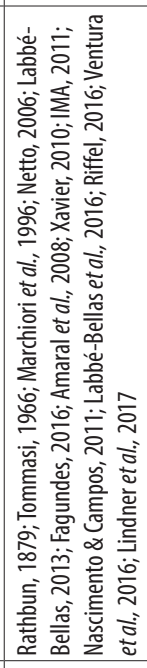 & 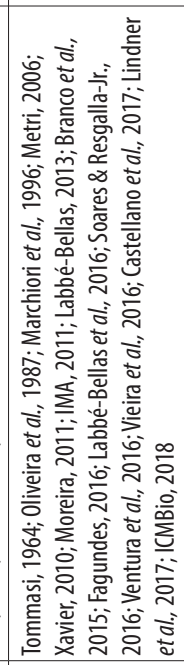 & 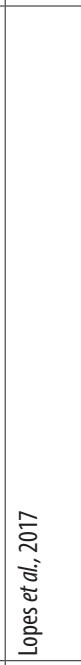 & 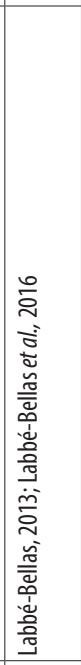 & 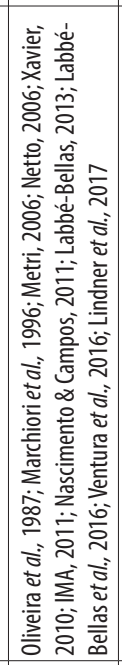 & 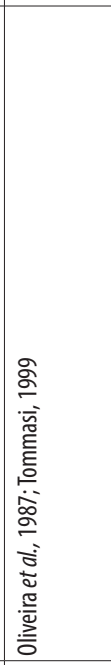 & 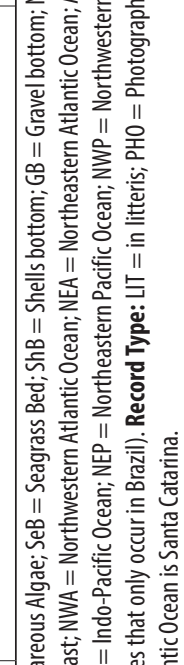 \\
\hline & \begin{tabular}{|l}
$\overline{\tilde{m}}$ \\
$\stackrel{\tilde{m}}{\tilde{m}}$
\end{tabular} & 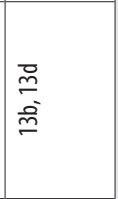 & & $\frac{a}{c}$ & 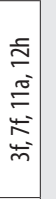 & 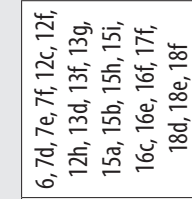 & 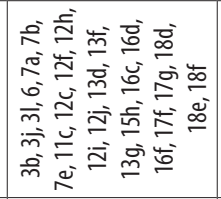 & 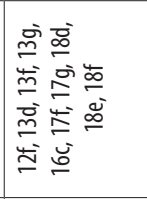 & 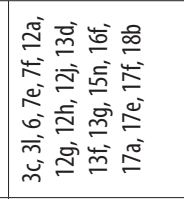 & $\breve{\dddot{m}}$ & 요 & 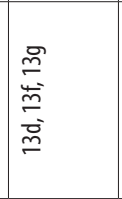 & 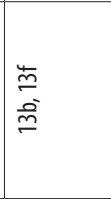 & 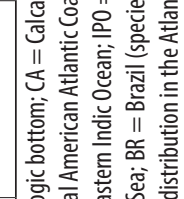 \\
\hline & 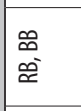 & 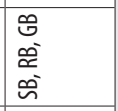 & & 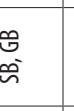 & $\mid \begin{array}{l}\mathscr{\sim} \\
\stackrel{\alpha}{\approx}\end{array}$ & $\approx$ & $\approx$ & $\approx$ & ఇ & 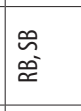 & 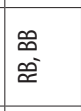 & 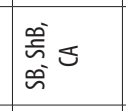 & $\begin{array}{l}\otimes \\
\ddot{n}\end{array}$ & 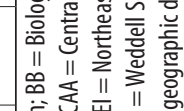 \\
\hline$z$ & 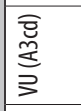 & 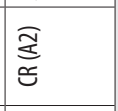 & & 岁 & 岁 & 㞫 & 㞱 & অ & 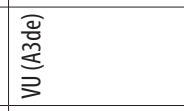 & 㞫 & 㞫 & 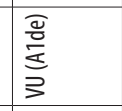 & 㞫 & 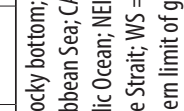 \\
\hline & 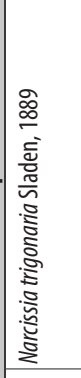 & 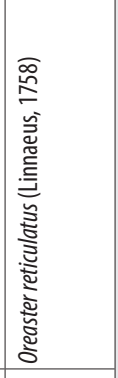 & & 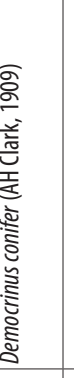 & 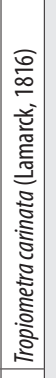 & 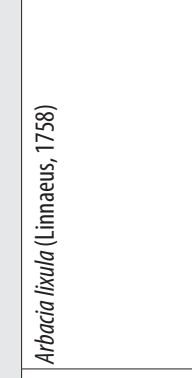 & 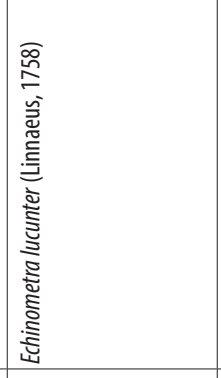 & 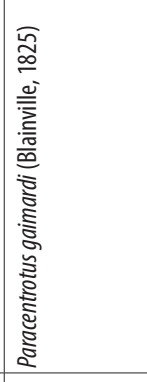 & 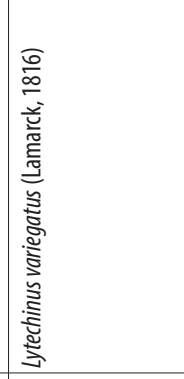 & 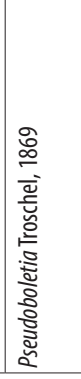 & 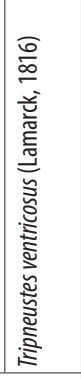 & 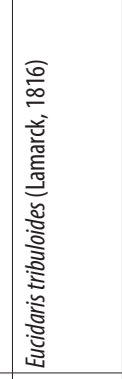 & 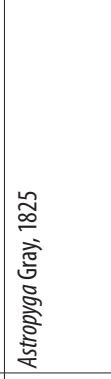 & 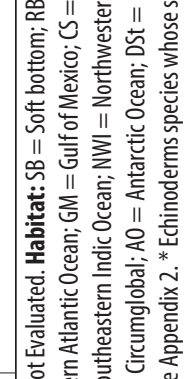 \\
\hline & 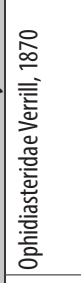 & 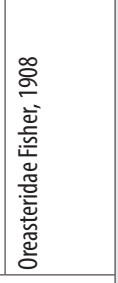 & & 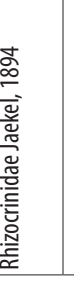 & 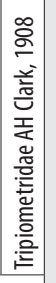 & 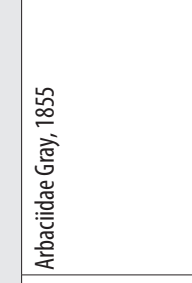 & 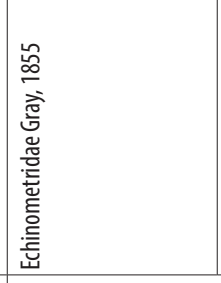 & 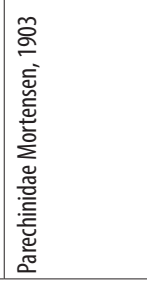 & 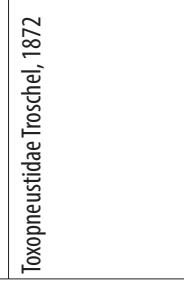 & & & 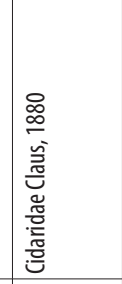 & 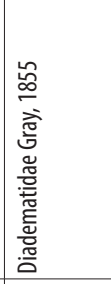 & 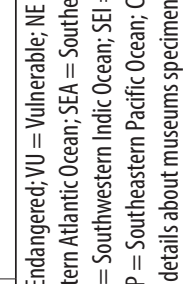 \\
\hline & & & 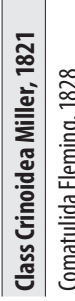 & 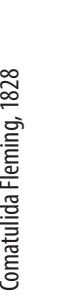 & & 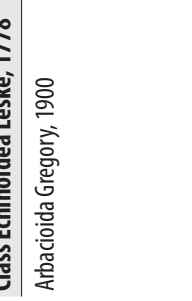 & 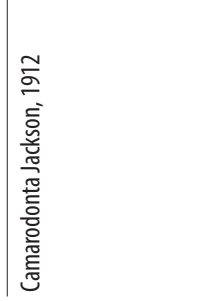 & & & & & 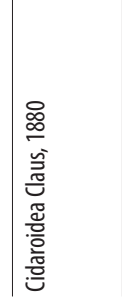 & 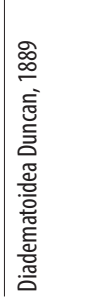 & 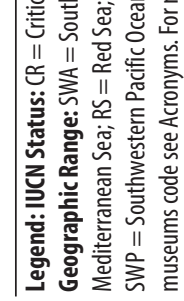 \\
\hline
\end{tabular}




\begin{tabular}{|c|c|c|c|c|c|c|c|c|c|c|c|c|c|c|c|c|c|c|}
\hline 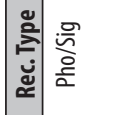 & $\Xi$ & $\begin{array}{l}\frac{0}{\bar{n}} \\
\frac{0}{2} \\
\frac{0}{2}\end{array}$ & $\begin{array}{l}\frac{0}{\bar{n}} \\
\frac{0}{2}\end{array}$ & & 新 & 을 & $\begin{array}{l}\frac{0}{\bar{n}} \\
\frac{0}{2} \\
\frac{0}{2}\end{array}$ & 䓂 & 홀 & 言 & 흘 & 言 & $=\frac{\bar{z}}{\frac{5}{2}}$ & 홍 & 言 & 을 $\frac{\bar{z}}{2}$ & 올 & 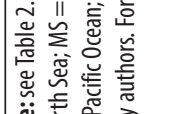 \\
\hline 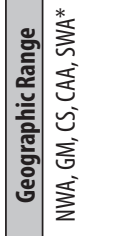 & 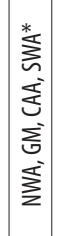 & 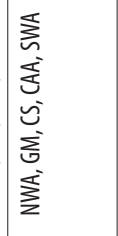 & 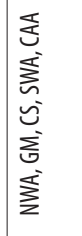 & 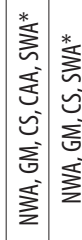 & 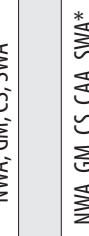 & 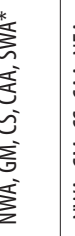 & 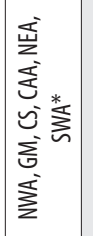 & 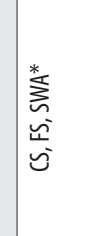 & 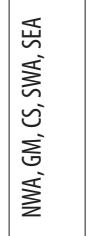 & 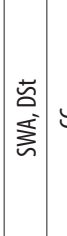 & অ & 离 & 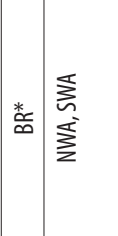 & $\stackrel{*}{\stackrel{*}{\infty}}$ & 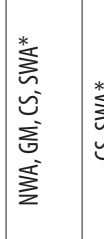 & 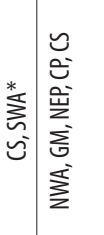 & 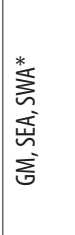 & 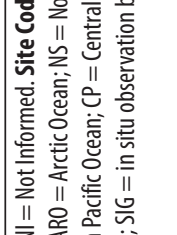 \\
\hline 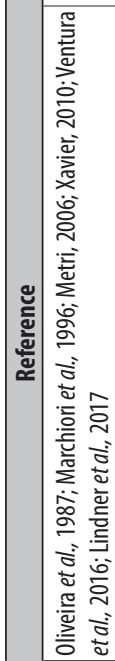 & 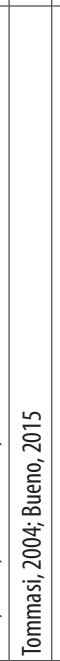 & 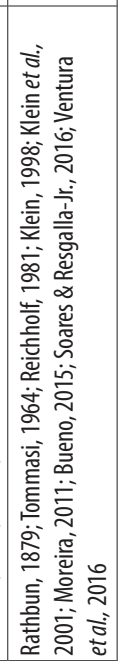 & 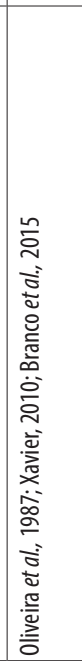 & 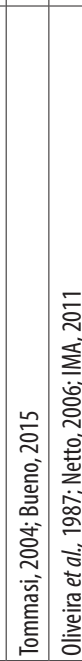 & 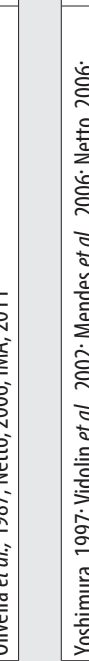 & 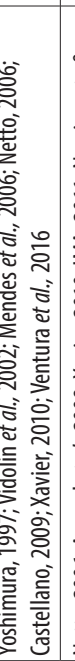 & 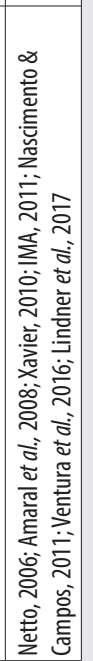 & 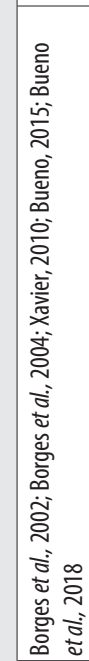 & 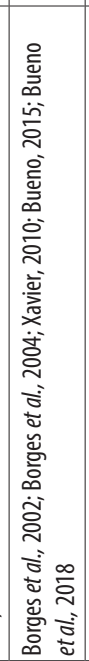 & 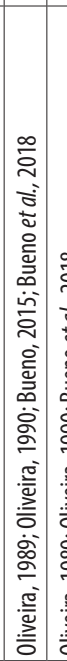 & 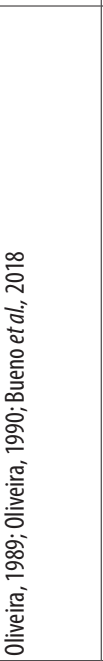 & 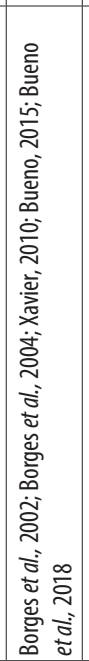 & 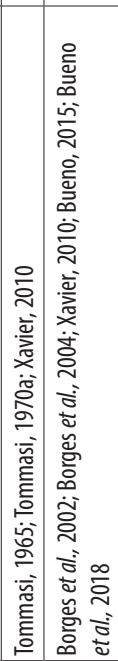 & 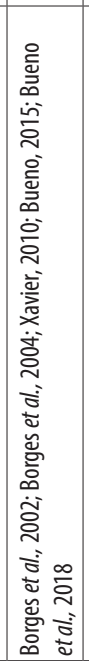 & 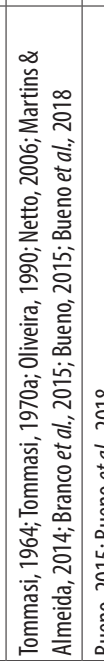 & 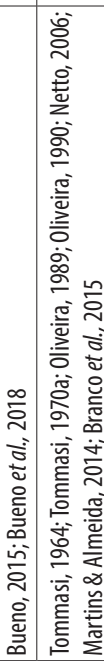 & 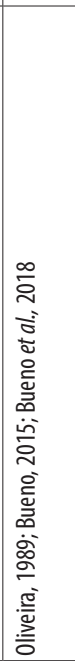 & 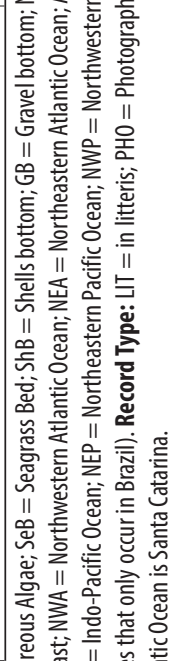 \\
\hline 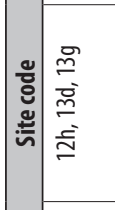 & $\begin{array}{l} \\
\stackrel{\Xi}{=} \\
\stackrel{\Xi}{\Xi} \\
=\end{array}$ & 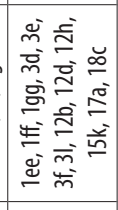 & 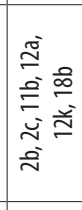 & $\Rightarrow \stackrel{2}{=}$ & 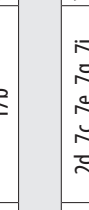 & 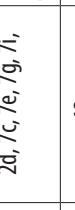 & $\stackrel{\widetilde{m}}{\mathscr{m}}$ & 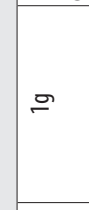 & $\underline{\underline{z}}$ & 2 & $\stackrel{\Xi}{\sigma}$ & $\begin{array}{l}0 \\
\frac{0}{50} \\
\underline{5}\end{array}$ & 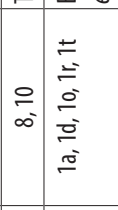 & 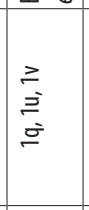 & 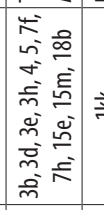 & 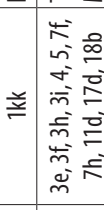 & 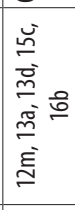 & 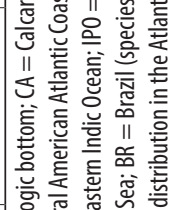 \\
\hline 莺 & $\begin{array}{l}\ddot{0} \\
\ddot{n} \\
\tilde{n}\end{array}$ & $\cong$ & 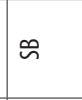 & $\begin{array}{ll}\infty & \infty \\
\tilde{n} & \curvearrowleft\end{array}$ & & 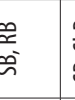 & 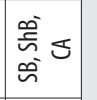 & 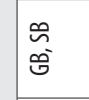 & 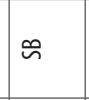 & $\cong$ & 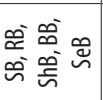 & $\begin{array}{l}\stackrel{9}{0} \\
\check{n}\end{array}$ & 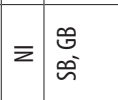 & 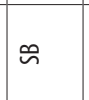 & 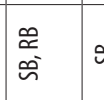 & $\rightsquigarrow$ & $\begin{array}{l}\infty \\
\ddot{n} \\
\tilde{n}\end{array}$ & \\
\hline$\underline{\underline{z}}$ & $\mathbf{z}$ & $\stackrel{\mathrm{z}}{\mathbf{z}}$ & 㞫 & 岁 & 岂 & $\vec{z}$ & $\begin{array}{l}\frac{\bar{g}}{\bar{c}} \\
\frac{5}{3}\end{array}$ & 亗 & 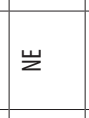 & $\vec{z}$ & 亗 & 岀 & 岁岸 & 㞫 & 岁 & 岁 & 亗 & \\
\hline 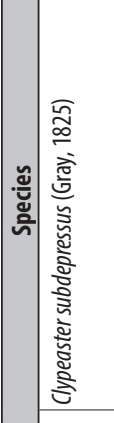 & 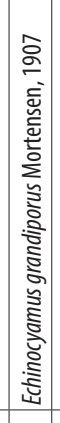 & 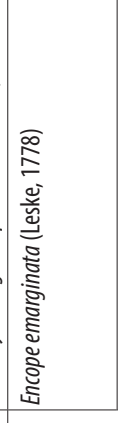 & 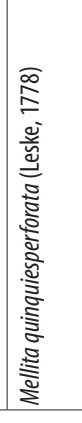 & 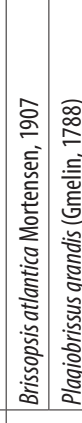 & 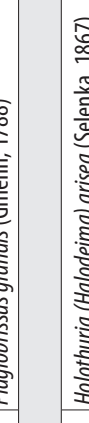 & 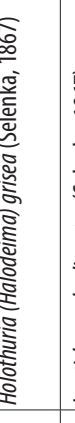 & 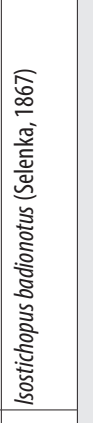 & 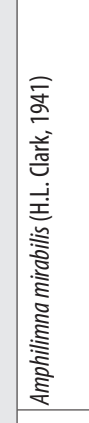 & 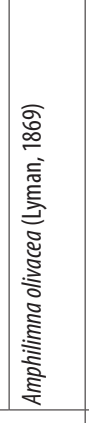 & 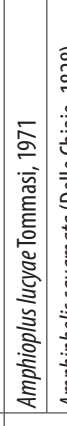 & 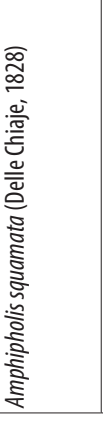 & 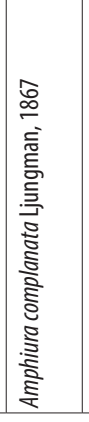 & 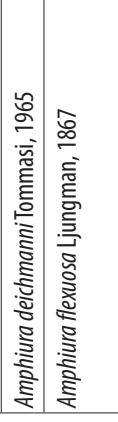 & 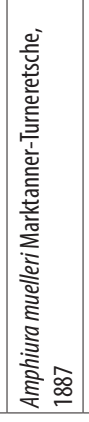 & 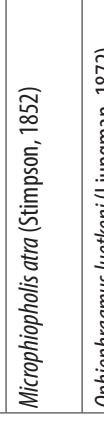 & 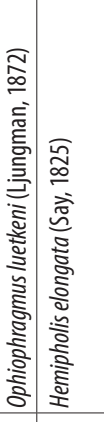 & 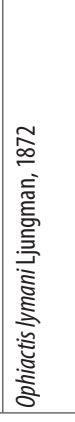 & 㣢 \\
\hline |c & 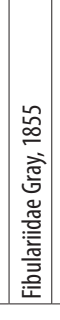 & 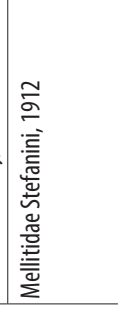 & & 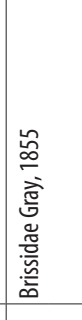 & 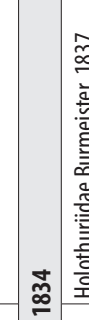 & 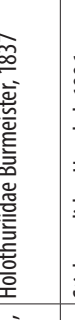 & 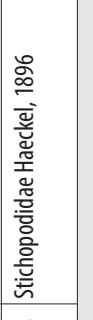 & 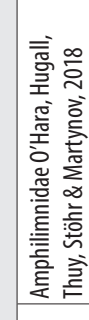 & & 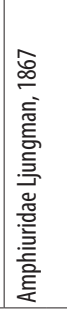 & & & & & & 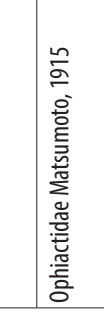 & & \\
\hline 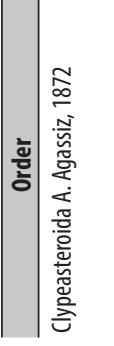 & & & & 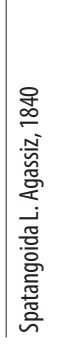 & 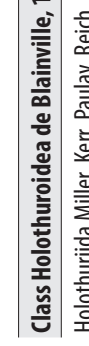 & 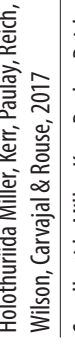 & 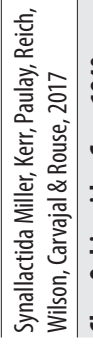 & 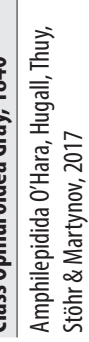 & & & & & & & & & & 定 \\
\hline
\end{tabular}




\begin{tabular}{|c|c|c|c|c|c|c|c|c|c|c|c|c|c|c|c|c|c|c|c|c|}
\hline & 言 & 홀 & 妾 & 产 & 흘 & 홍 & $\begin{array}{l}\text { 产 } \\
\text { 옴 }\end{array}$ & 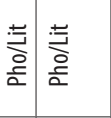 & 章 & 흘 & 产 & 䓂 & $\begin{array}{l}\text { 产 } \\
\text { 옴 }\end{array}$ & 홓 $\frac{5}{\frac{3}{2}}$ & 䇏 & $\Xi$ & $\frac{5}{a}$ & 옴 & & 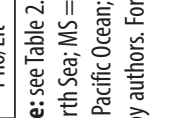 \\
\hline & & 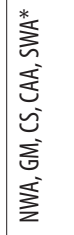 & 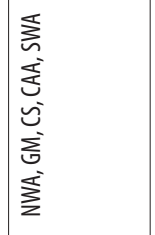 & $\approx$ & $\cong$ & 䓂 & 旅 & 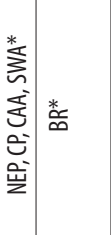 & $\stackrel{*}{\infty}$ & $\begin{array}{l}\text { 訔 } \\
\text { o }\end{array}$ & 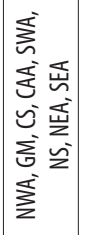 & 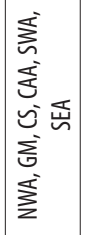 & 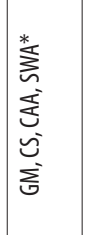 & 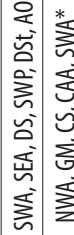 & 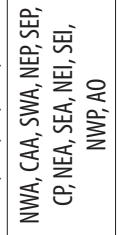 & 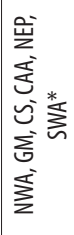 & 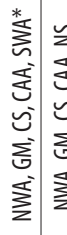 & 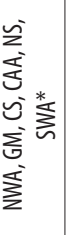 & & 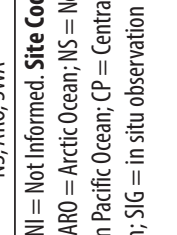 \\
\hline & 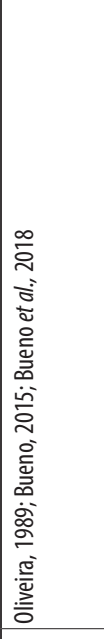 & 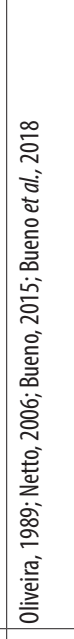 & 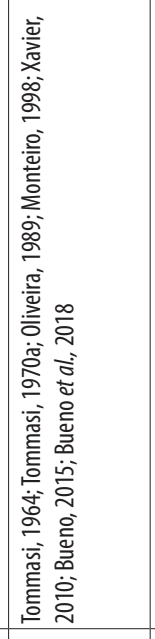 & 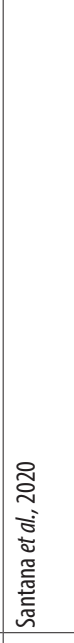 & 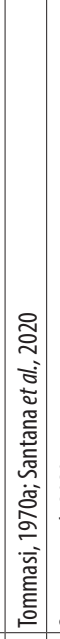 & 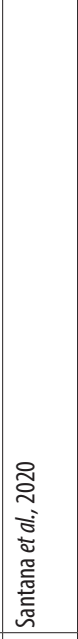 & 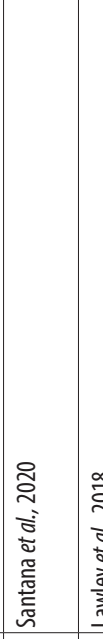 & 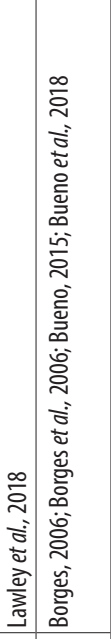 & 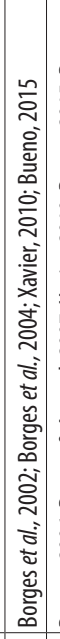 & 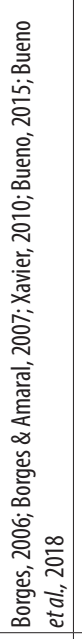 & 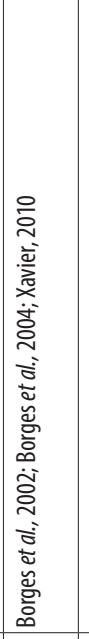 & 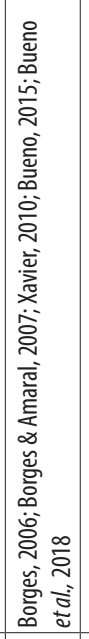 & 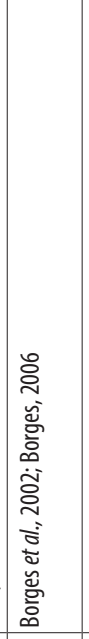 & 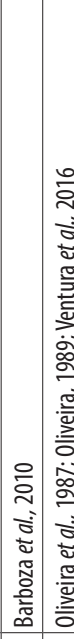 & 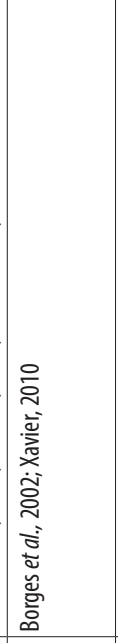 & 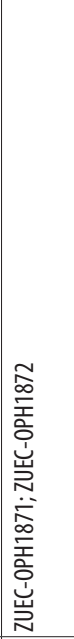 & 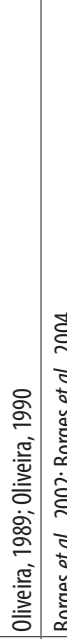 & 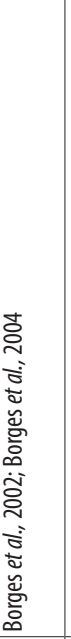 & & 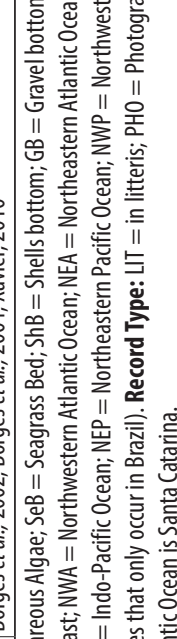 \\
\hline & 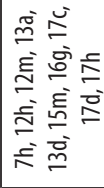 & 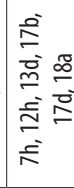 & 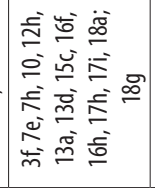 & $\stackrel{\overbrace{}}{\circ}$ & $\grave{m}$ & 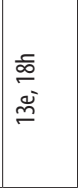 & $\stackrel{\Phi}{\infty}$ & 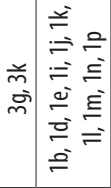 & 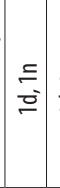 & 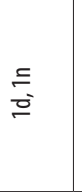 & Ð & 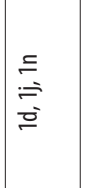 & $\simeq$ & 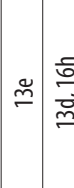 & 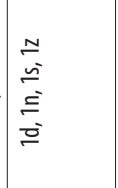 & $\stackrel{x}{\rightleftarrows}$ & 怘 $=$ & 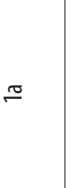 & $\frac{0}{\square}$ & 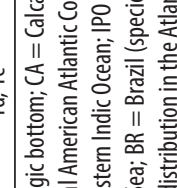 \\
\hline 离 & 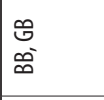 & 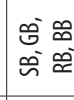 & 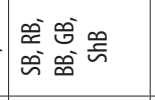 & $\bar{z}$ & \begin{tabular}{|l|}
$\infty$ \\
$\approx$ \\
$\tilde{n}$
\end{tabular} & $\bar{z}$ & $\bar{z}$ & 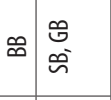 & $\begin{array}{l}: \\
\tilde{5} \\
\tilde{n}\end{array}$ & $\begin{array}{l}\ddot{9} \\
\ddot{~}\end{array}$ & $\begin{array}{l}\ddot{9} \\
\ddot{n}\end{array}$ & $\begin{array}{l}\ddot{\Xi} \\
\ddot{n}\end{array}$ & $\cong$ & 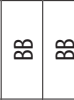 & $\begin{array}{l}\ddot{\Xi} \\
\ddot{n}\end{array}$ & $\bar{z}$ & 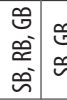 & 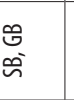 & $\begin{array}{l}m \\
\stackrel{m}{n}\end{array}$ & 象 \\
\hline s. & 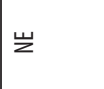 & 岀 & 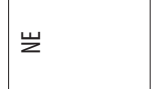 & 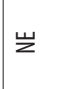 & 岁 & 亗 & 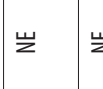 & 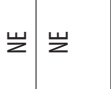 & $\vec{z}$ & 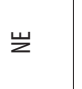 & 岁 & 岀 & 岁 & $\vec{z}$ & 岀 & 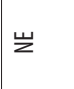 & $\vec{z}$ & 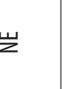 & $\vec{z}$ & \\
\hline & 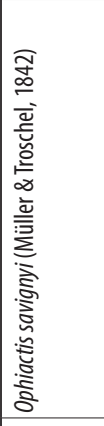 & 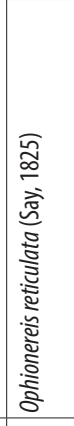 & 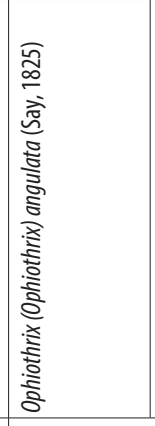 & 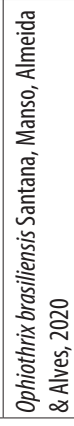 & 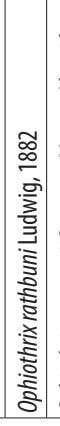 & 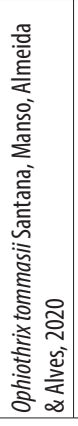 & 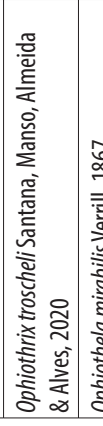 & 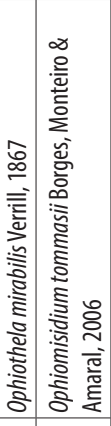 & 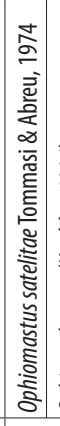 & 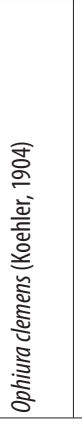 & 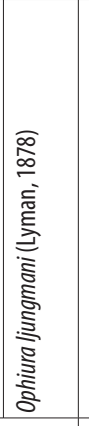 & 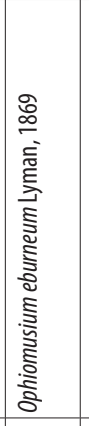 & 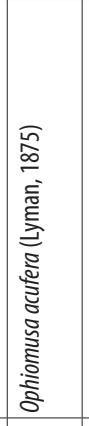 & 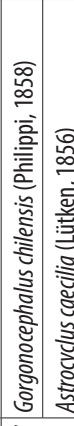 & 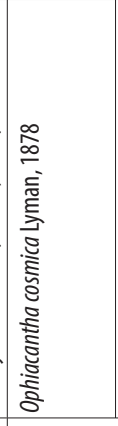 & 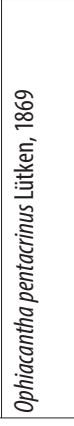 & 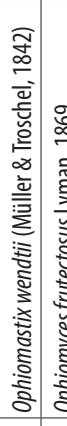 & 离 & & 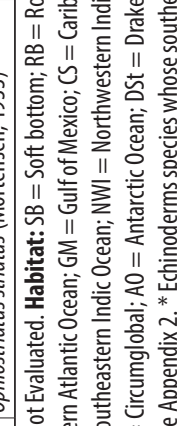 \\
\hline & & 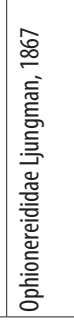 & 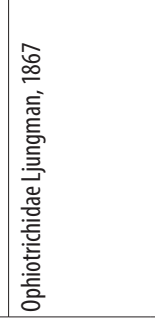 & & & & & 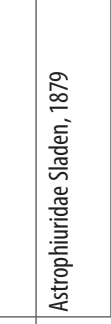 & 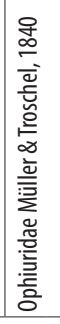 & & & 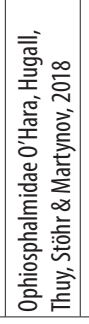 & 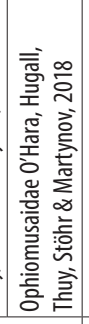 & 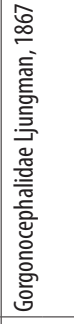 & 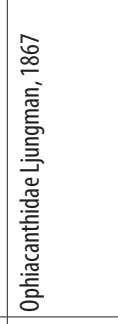 & & 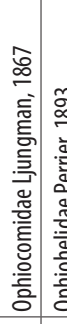 & 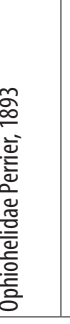 & & 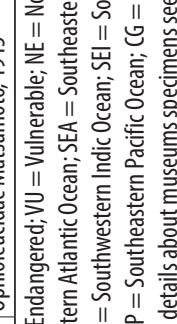 \\
\hline & & & & & & & & 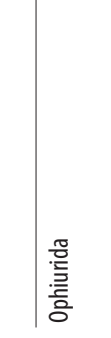 & & & & & & 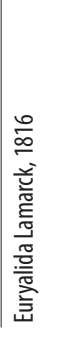 & 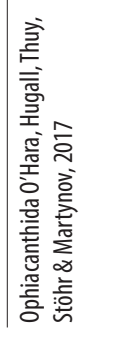 & & & 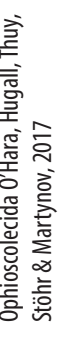 & & 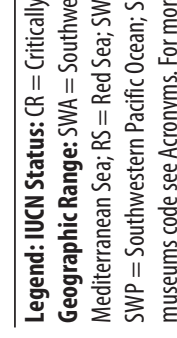 \\
\hline
\end{tabular}



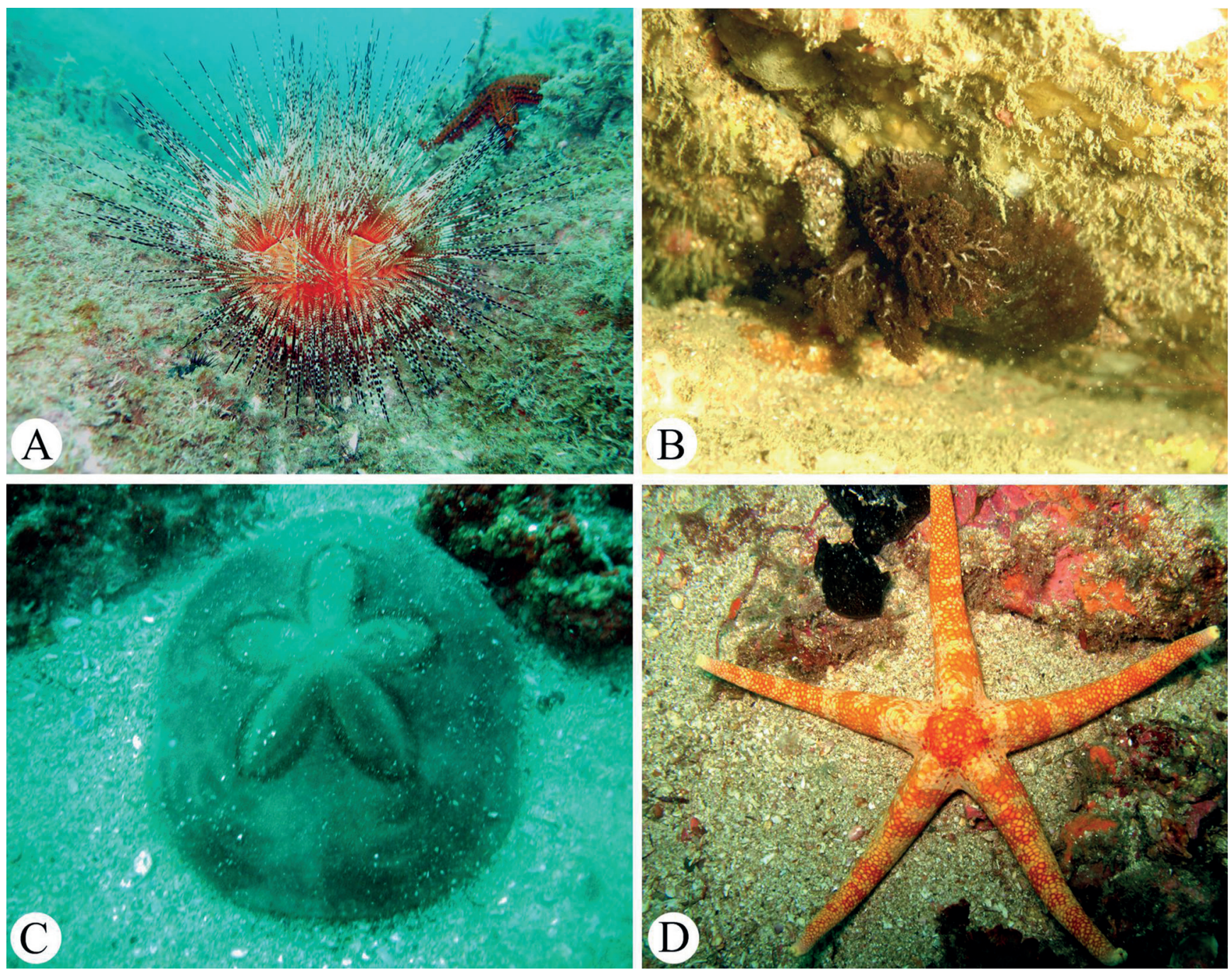

Figure 1. Some echinoderm species from Santa Catarina. (A) Astropyga sp. (Arvoredo Island; Photo: Jéssica Link), (B) Holothuria (Halodeima) grisea (Arvoredo Island; Photo: Thiago Fiuza), (C) Clypeaster subdepressus (Xavier Island; Photo: Nataly Slivak), (D) Narcissia trigonaria (Xavier Island; Photo: João Luís Carraro).
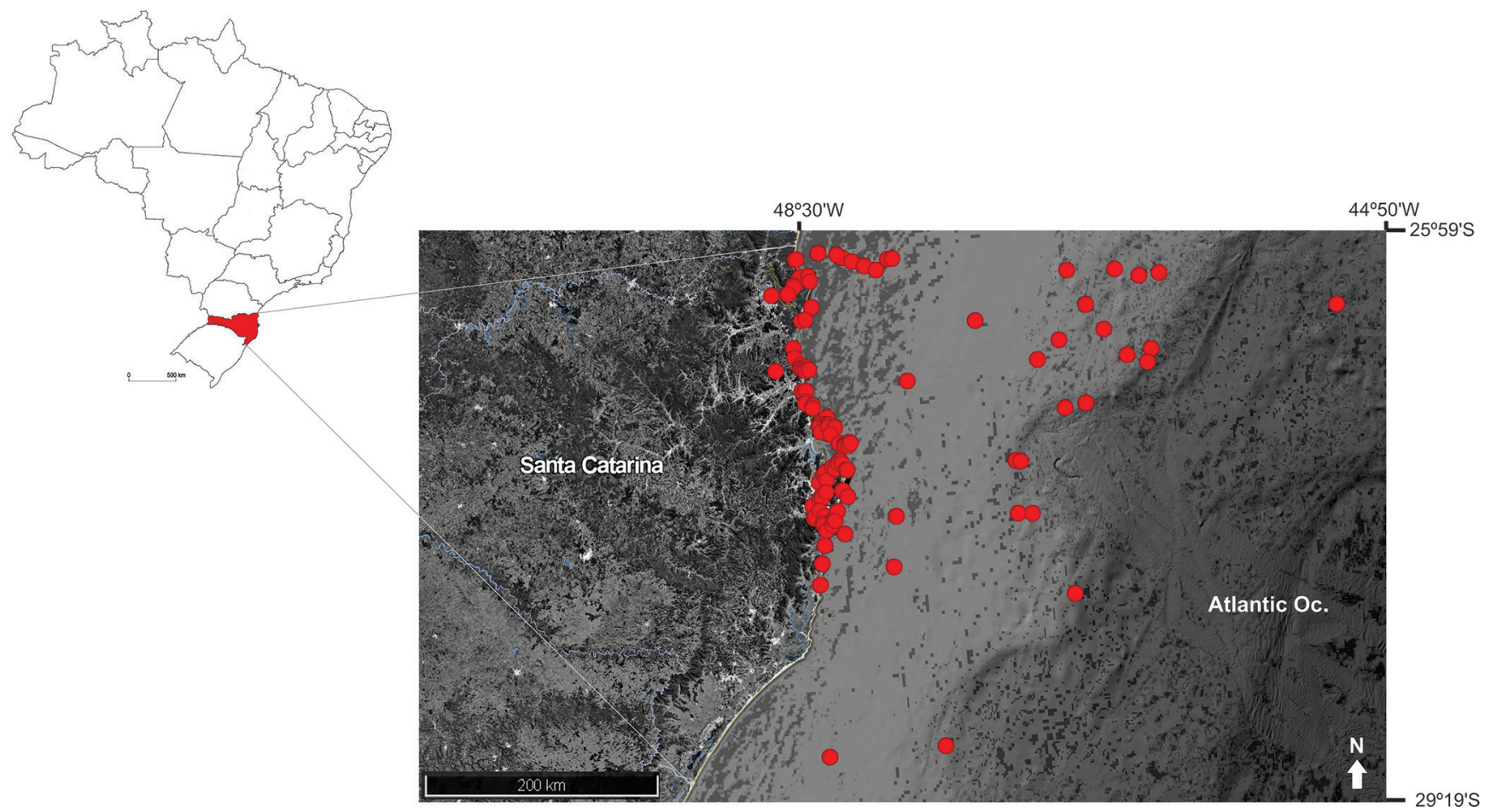

Figure 2. Map of Santa Catarina State, Brazil, showing the record sites of echinoderm species (red circles). 


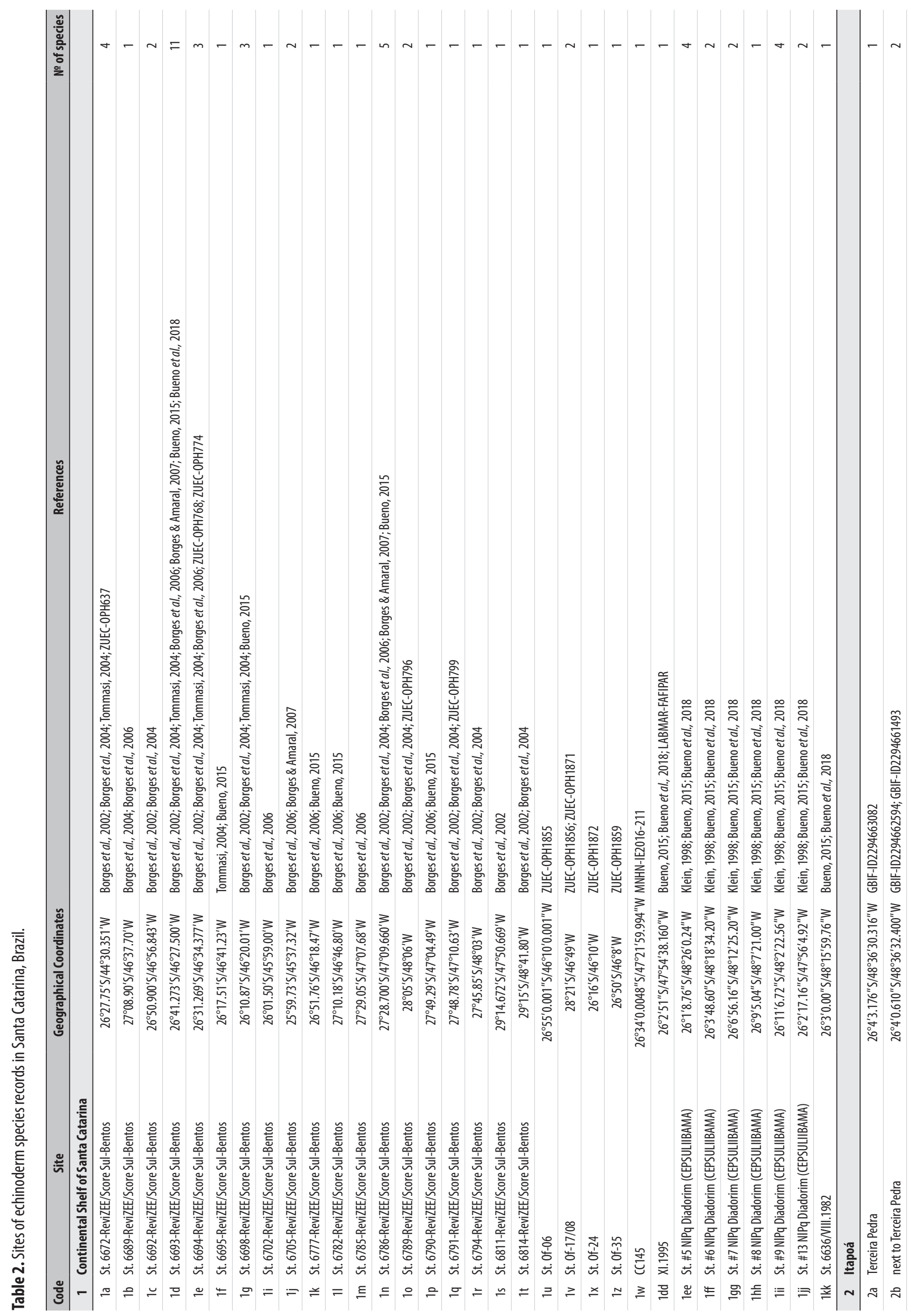




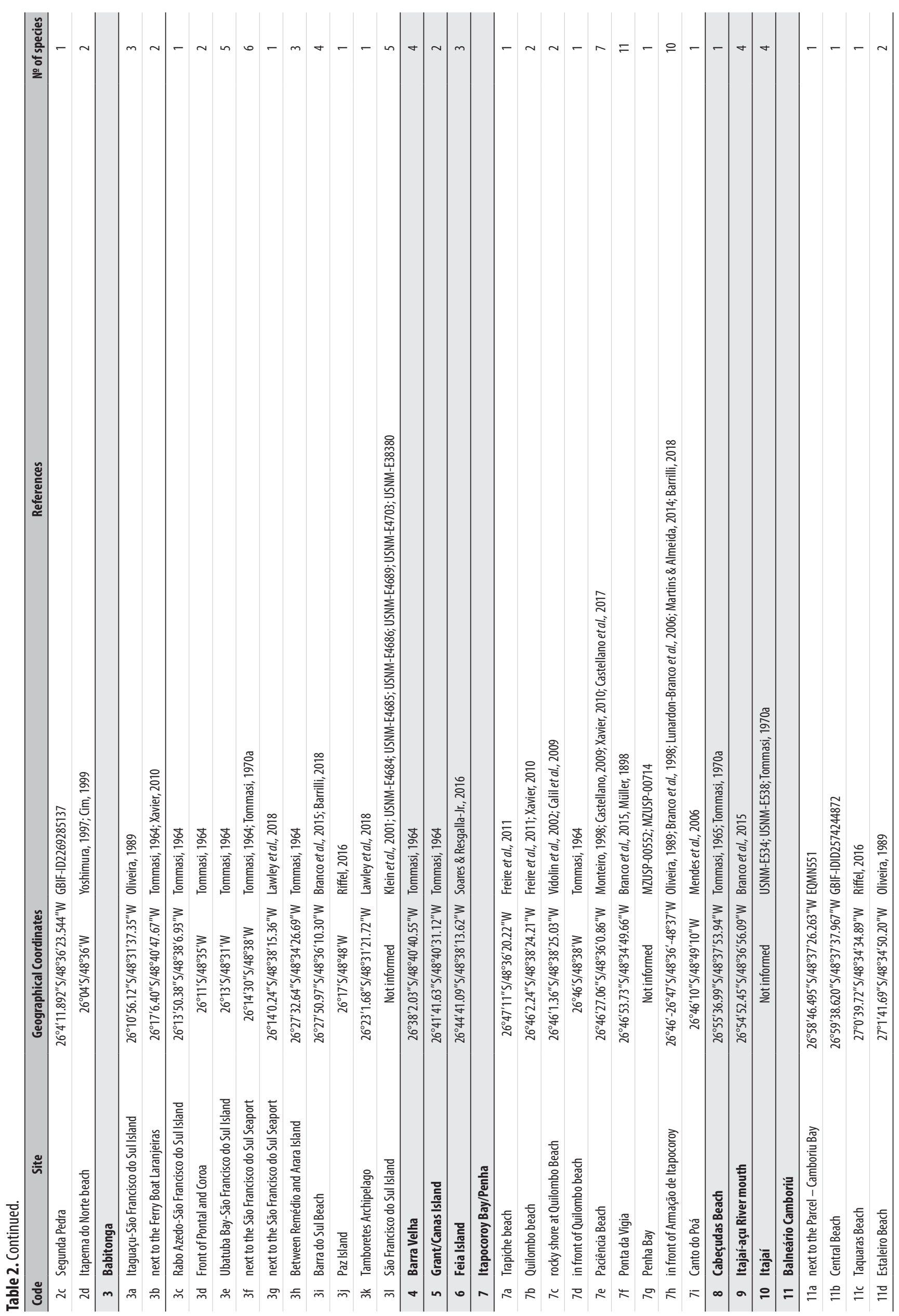




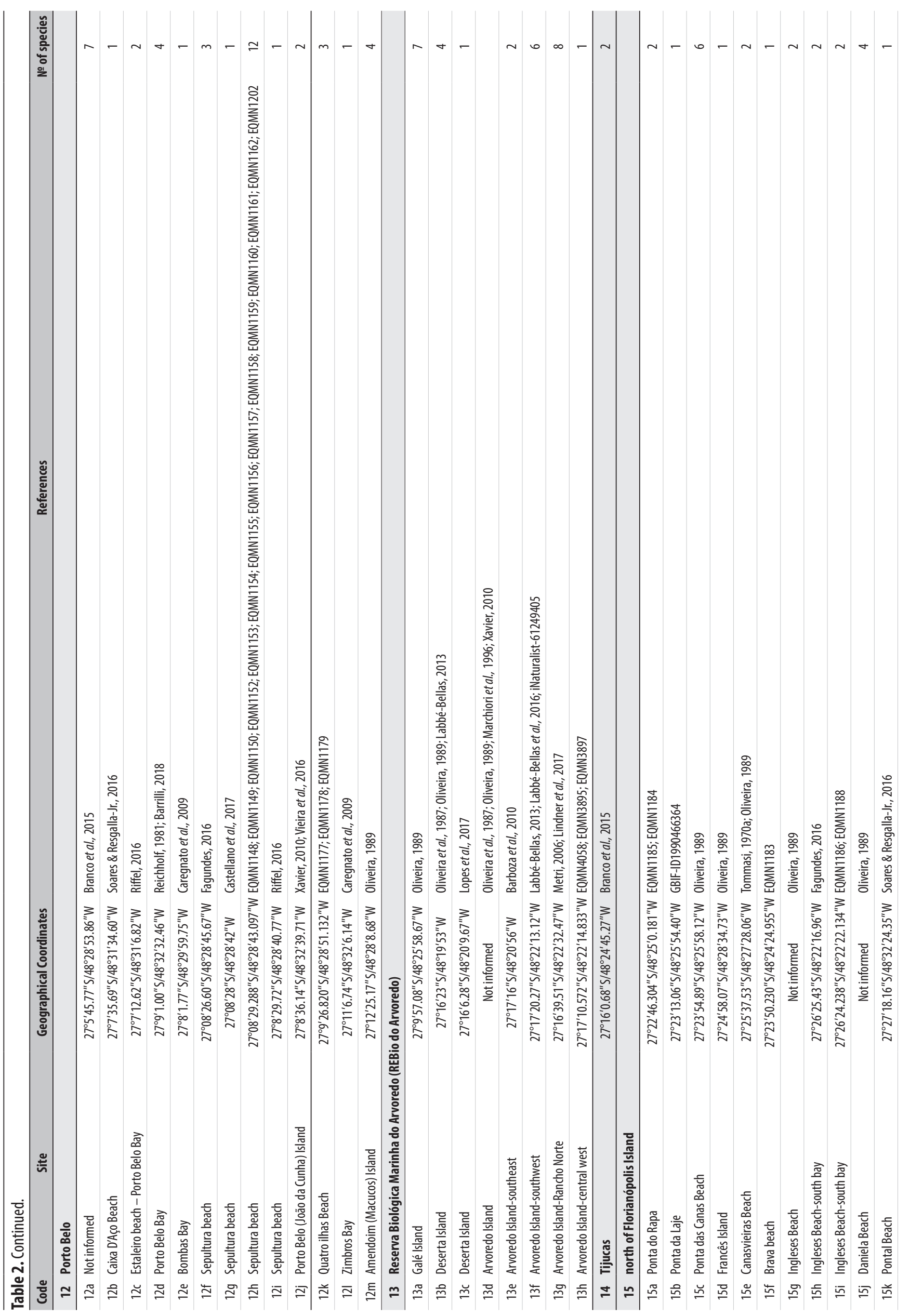




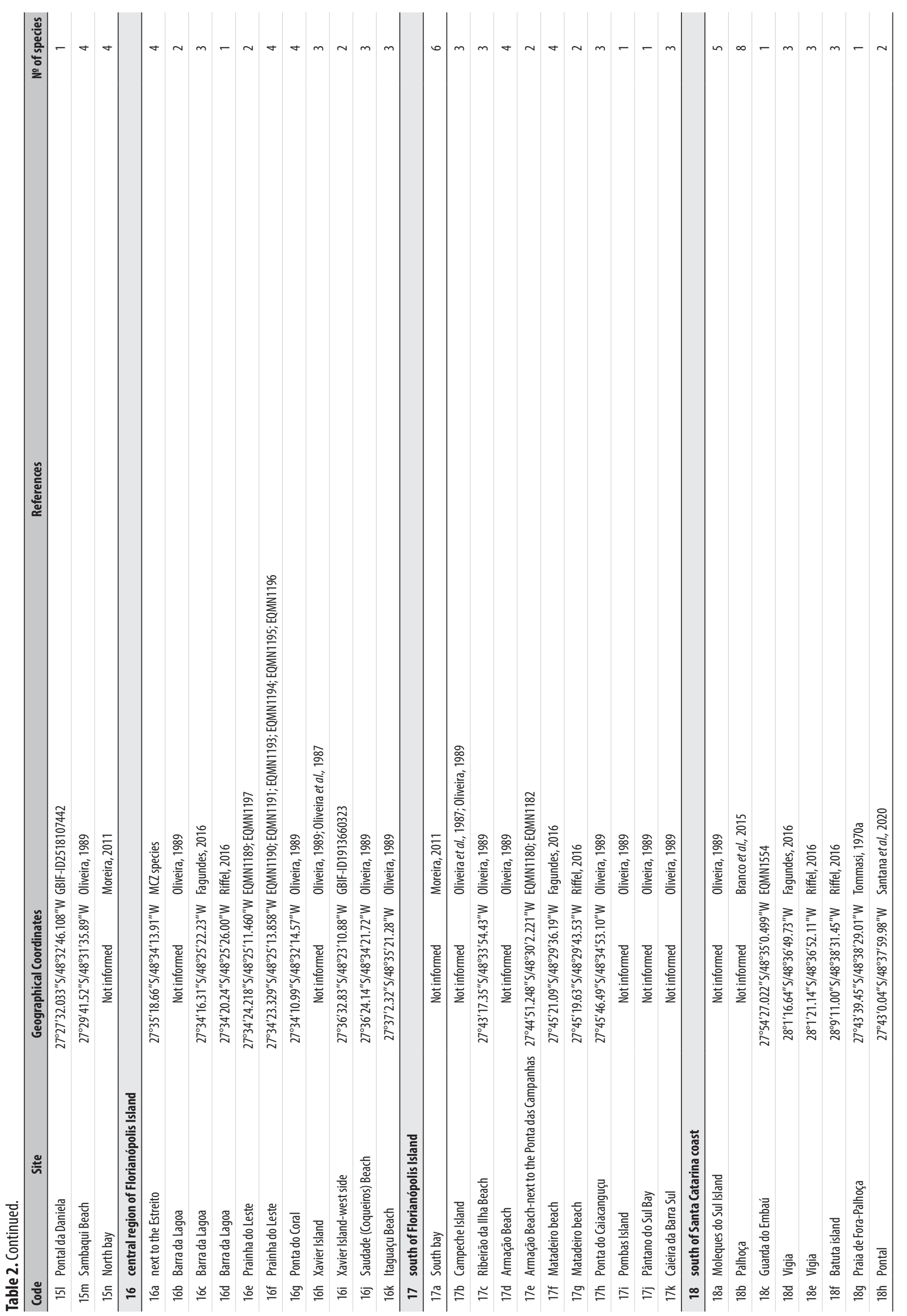




\section{Echinoderm distribution along Santa Catarina coast}

Of the 134 sites where echinoderm species were recorded (Fig. 2), the shallow continental shelf and the Arvoredo area concentrated the highest number of species. The continental shelf of Santa Catarina was mainly explored by the ReviZEE program (Living Resources in the Exclusive Economic Zone). The program provided the discovery of new marine species for Brazil, especially those inconspicuous and difficult to be sampled, such as ophiuroids (Borges et al., 2002, 2006). In this study, we observed that most of the ophiuroids registered for Santa Catarina came from the ReviZEE program (45\%). Due to mobility, diversity of eating habits and small size, ophiuroids are able to explore habitats often unattainable by other echinoderms (Hyman, 1955; Bueno et al., 2018).

Part of Arvoredo Island is within the Arvoredo Marine Biological Reserve, a conservation unit that also includes Galé and Deserta Island, and Calhau de São Pedro (Segal et al., 2017). The echinoderm records reported herein are mainly from the north and center-north coast of Santa Catarina ( 26 species), where the Arvoredo Marine Reserve is located. Rocky shores and coastal islands characterize this coastal region, environments suitable for several echinoderm species. In addition, most marine research institutions are located in the center-north region of Santa Catarina, and thus may contribute to the largest sample effort in this area. In contrast, the results revealed a lower number of echinoderm records in the southern region of Santa Catarina. Sandy beaches and dunes characterize the southern coast of Santa Catarina, where marine and wind processes predominate. The echinoderm species reported in the literature for the southern coastal region were the epibenthic sea biscuits, Encope emarginata and Melitta quinquiesperforata (Tavares, 1996; Brustolin, 2013).

These animals live under sandy and/or muddy substrate and require different sampling techniques, such as fishing nets and trawls. The lower number of echinoderms recorded between $27^{\circ} 50^{\prime} \mathrm{S}$ and $29^{\circ} 20^{\prime} \mathrm{S}$ latitudes can indicate that the echinoderm diversity presented in this study may be underestimated due to the absence of further studies and/or appropriate sampling methods.

Ophiuroidea was the most representative class, with 33 species recorded (50\%). Some authors also observed similar results for the northeast region of Brazil, such as $50 \%$ ophiuroid records in Bahia state (Alves \& Cerqueira, 2000; Magalhães et al., 2005), 40\% in Paraíba (Gondim et al., 2008) and $42 \%$ in Pernambuco (Lima \& Fernandes, 2009). In Paraná state, southern Brazil, ophiuroid diversity (34 species) was similar to this study, comprising $55 \%$ of the echinoderm fauna (Bueno et al., 2018). Ophiuroidea representativeness is a pattern on the Brazilian coast, comprising 153 extant species $(43.7 \%$ of 347 species of echinoderms) (Ventura et al., 2013). Asteroidea and Echinoidea were the second and third most representative classes at Santa Catarina (22.7\% each) followed by Holothuroidea and Crinoidea ( $2.6 \%$ each). The starfish Asterina stellifera and Echinaster (Othilia) brasiliensis occurred in at least 29 coastal sites, and the sea urchin Echinometra lucunter and Arbacia lixula, in 25 and 21, respectively (Table 1). Our results also reveal that Santa Catarina is the southernmost record for 40 species, that is, about $60 \%$ of the species recorded (Table 1; see Geographic Range column). In addition, 12 of the recorded species listed in this study (17\%) are endemic to the Brazillian coast, such as two ophiuroid species recently described, Ophiothrix tommasii Santana, Manso, Almeida \& Alves, 2020 and Ophiothrix troscheli Santana, Manso, Almeida \& Alves, 2020. New species recorded recently (Santana et al., 2020) and new occurrences (Labbé-Bellas et al., 2016; Lopes et al., 2017) show that there is still much to learn about the echinoderm fauna of Santa Catarina.

\section{Taxonomic information}

In this study, we provided the updated species names for some ophiuroids, such as Ophiomusa acufera (before named Ophiomusium acuferum) and Ophiomastix wendtii (before named Ophiocoma wendtii Müller \& Trsochel, 1842 and Ophiocoma riisei Lütken, 1856) (O'Hara et al., 2018a, b). As presented above, in Brazil ophiuroids are one of the most abundant and studied groups of the Echinodermata macrofauna (Ventura et al., 2013). Consequently, the systematics of ophiuroids is currently in flux (Albuquerque et al., 2001; Borges et al., 2002, Borges \& Campos, 2011; Borges \& Amaral, 2007; Gondim et al., 2010, 2014, 2015; Manso et al., 2014; Alitto et al., 2020; Santana et al., 2020), and with the increase in molecular data, more species can be expected to be discovered.

In Santa Catarina, the starfish Echinaster (Othilia) brasiliensis was identified in 29 sites, recorded by 14 scientific references (Table 1). In Brazil, three Othilia species have been recorded, Othilia brasiliensis (Tommasi, 1970b; Hopkins et al., 2003; Netto et al., 2005; Lima \& Fernandes, 2009; Gondim et al., 2011, 2020; Miranda et al., 2012; Ventura et al., 2013; Alitto et al., 2016), Othilia guyanensis (Hopkins et al., 2003; Mariante et al., 2010; Ventura et al., 2013) and Othilia echinophorus (Tommasi, 1970b; Alves \& Cerqueira, 2000; Hopkins et al., 2003; Gondim et al., 2008, 2011, 2020; Lima \& Fernandes, 2009; Miranda et al., 2012; Ventura et al., 2013). However, according to the phylogenetic study by Lopes et al. (2016), O. brasiliensis and $O$. guyanensis should be synonymized, and the status of O. echinophorus should be reviewed; the authors emphasize the large morphological variability and phenotypic plasticity of the genus Echinaster, and support the raise of the genus Othilia, previously demoted to subgenus (Clark \& Tortonese, 1986). Morphological (Fontanella \& Hopkins, 2003) and molecular data (Lopes et al., 2016) have shown that Othilia is a monophyletic group and that the genus Echinaster is closer and more related to Henricia Gray 1840 than to Othilia.

Two echinoderm genera, in the present study, are not identified at the species level, the equinoids Astroypga and Pseudoboletia. The genus Astropyga is recorded for Brazil as Astropyga nuptialis, described for the state of São Paulo (Tommasi, 1958), and as Astropyga magnifica A.H. Clark, 1934 for Santa Catarina and Pernambuco 
(Oliveira et al., 1987; Lima \& Fernandes, 2009). Oliveira et al. (1987) recorded $A$. magnifica for Deserta Island (Arvoredo Marine Reserve) at $12 \mathrm{~m}$ depth. Recently, echinoderm researcher Cesar Cordeiro identified this genus at Arvoredo Island (ReBio Arvoredo) (Photo: iNaturalist - 61249405). However, as we did not have access to this material, we chose to keep it at genus level, and we suggest a taxonomic study to identify which of the two Astropyga species occur in Santa Catarina.

Lopes etal. (2017) recorded the genus Pseudoboletiasp. at Deserta Island, Santa Catarina and Cabo Frio Island, Rio de Janeiro. The molecular results differentiated three species of this genus, two from the Indo-Pacific ( $P$. indiana and P. maculata) and one from the Atlantic (Pseudoboletia sp. from São Tome and Brazil). Martins et al. (2018) referred the specimens from the Trindade Island, as P. maculata, whose morphological characteristics are the same as those of $P$. maculata from the Indo-Pacific, but different from those recorded by Lopes et al. (2017) in Rio de Janeiro and Santa Catarina.

In this study, we adopted a method of taxonomic reliability (see "Record Type" in Table 1). Direct access to the documented literature material is not always possible, but it is important to validate this information. Although we included data from unpublished studies, these species records were included herein when also presenting photographs of the reported species. Most studies are specific publications on echinoderms (about 90\%), carried out by experts in the group. We emphasize the importance of this literature for this checklist and for future studies on local biodiversity and ecology.

During this work, one ophiuroid species was reported for the first time in Santa Catarina, Ophiacantha pentacrinus. This specimen was identified by $\mathrm{Dr}$. Michela Borges, ophiuroids specialist, and deposited in the Museu de Zoologia da UNICAMP (Coleção de Ophiuroidea) (UNICAMP Museum of Zoology (Collection of Ophiuroidea)), but was not previously reported in the literature. The species Ophiacantha cosmica and Ophiacantha pentacrinus have long been confused, taxonomically. Species identified as O. cosmica may actually be of this species or an O. pentacrinus (Tommasi, 1999). Even today, echinoderm specialists work with these two species in order to guarantee the correct identification. In addition to being a new record of this species in Brazil, Santa Catarina is the southernmost location for the distribution of $O$. pentacrinus.

\section{Habitat distribution}

Echinoderms inhabit all types of marine substrate (Ventura et al., 2016). Most species of ophiuroids are bottom dwellers on the sea floor, buried in mud or hidden in crevices and holes in rock or coral (Stöhr et al., 2008, 2012). In Santa Catarina, we observed that some ophiuroid species are epizoic, such as Ophiactis savignyi, which colonizes other ophiuroids species, and Astrocyclus caecilia, which was observed on Ellisella elongata (Pallas, 1766), a gorgonian species (Oliveira, 1989;
Stöhr et al., 2012). Starfishes occur in unconsolidated (sandy, muddy) and consolidated (rocks) substrates. All starfish species recorded in this work occur in soft botton. The species of the families Astropectinidae and Luidiidae are infaunal, that is, they spend most of their time under the substrate, such as Astropecten brasiliensis and Luidia senegalensis (Clark \& Downey, 1992). However, Echinaster (Othilia) brasiliensis, for example, lives on rocks, rhodolites and shells, as they are mainly sponge-eating animals (Fergunson, 1969).

Echinoids, such as Arbacia lixula, Echinometra lucunter and Tripneustes ventricosus, typically inhabit rocky coast, rocky bottoms and seagrass beds (Mah \& Blake, 2012; Tavares \& Borzone, 2015), the same substrate pattern is observed for these species at Santa Catarina coast. In Northern Brazil, these animals also occur in tidal pools, biogenic reefs, crevices and dead coral substrate (Santos et al., 2002; Lima et al., 2009; Labbé-Bellas et al., 2016).

Crinoids occur in all oceans and ranging from coastal to abyssal depths, such as Tropiometra carinata, a common species on the Brazilian coast (MacCord \& Duarte, 2002; Souto \& Martins, 2017). This species was usually observed by the first and second authors as attached to the lateral surface of rocks, such as illustrated by Ventura et al. (2016). Democrinus conifer was collected on the outer continental shelf, in Santa Catarina, through the Living Resources Program in the Exclusive Economic Zone (ReviZEE), Score Sul/Bentos (Tommasi, 2004). This species has been recorded for Rio de Janeiro, São Paulo and Paraná (Bueno et al., 2018), however little information about this species in Brazilian waters is available.

Two holothurians species are present in this study, Holothuria (Halodeima) grisea and Isostichopus badionotus. Mendes et al. (2006) reported Holothuria (Halodeima) grisea in Armação do Itapocoroy Bay with a clumped distribution on rocky bottoms, however in sandy bottom areas, there was a high frequency of isolated specimens (not clumped). This seems to be a pattern along the Brazilian coast (Alves \& Cerqueira, 2000; Miranda et al., 2012; Alitto et al., 2016; Leite-Castro et al., 2016). Isostichopus badionotus is an epibenthic holothurian widely distributed on shallow muddy, sandy and seagrass bed, but can also be found in coral reefs (ZacaríasSoto et al., 2013). In Santa Catarina, this species was observed in Arvoredo Island on sandy substrate, with different color patterns. There is little information about this species in Brazilian waters.

\section{Threatened species}

In this study, 16 threatened species were recorded (21\% of total) (IMA, 2011). Of the 16 starfish species recorded, six are on the endangered national fauna list (ICMBio, 2018). According to Ventura et al. (2013), accidental capture by shrimp trawlers is one of the main threats to echinoderms, especially for asteroids that live in sandy and muddy bottoms, such as Astropecten brasiliensis, Astropecten cingulatus, Luidia clathrata and Luidia senegalensis. In Santa Catarina, Branco et al. (2015) 
recorded 11 echinoderms species as bycatch in artisanal fishing for the seabob shrimp Xiphopenaeus kroyeri (Heller, 1862), that is, $68.75 \%$ of the total threatened echinoderm species in the Santa Catarina coast. In this context, knowledge about factors that affect the structure of communities in the areas in which trawling fisheries operate can be an important tool for the correct and sustainable management of fisheries (Barrilli, 2018).
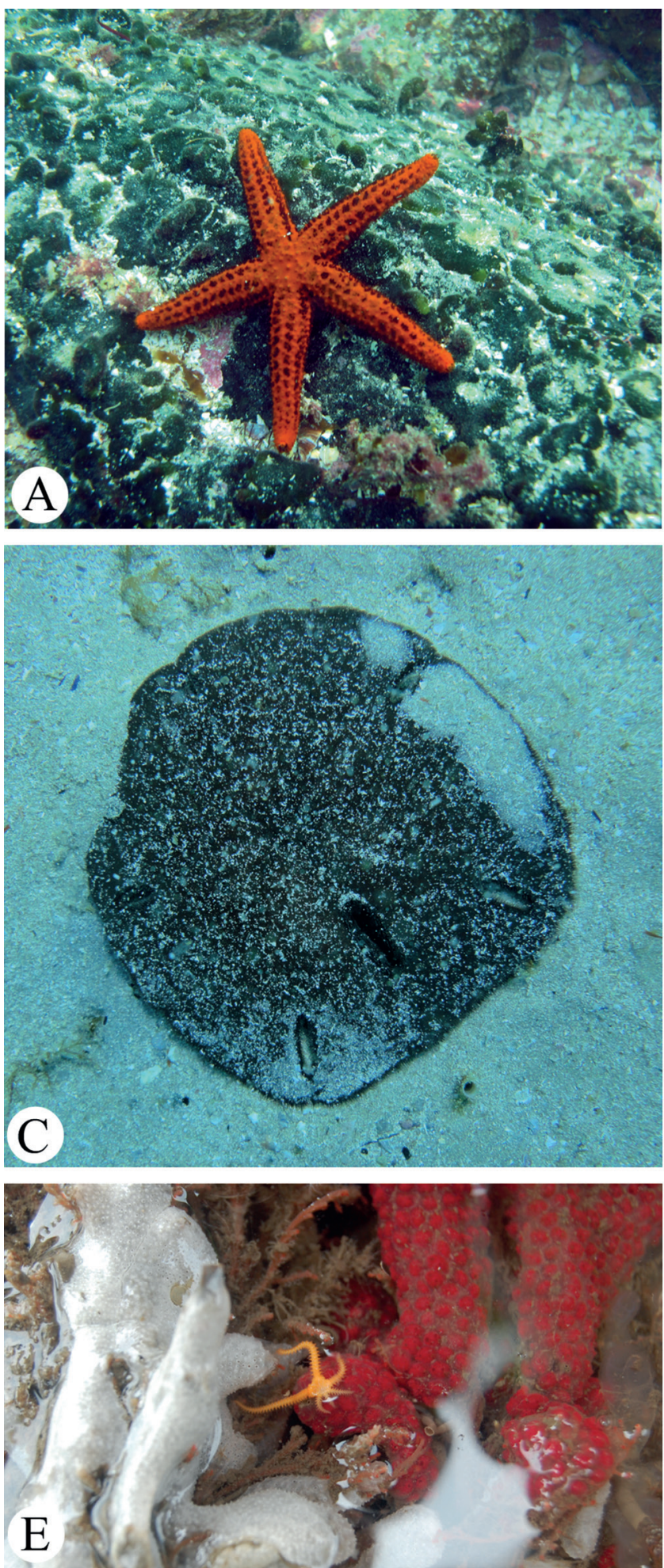

Other potential threats to echinoderms in Santa Catarina are aquarium trade and commercial fishing for souvenirs and decorative items that affect starfishes such as Oreaster reticulatus and Asterina stellifera, which are captured and marketed to tourists (Ventura et al., 2013; Branco et al., 2015; Souto \& Martins, 2017). In addition, although the echinoderm exploration for food purpose in Brazil is still small, the echinoid Echinometra lucunter
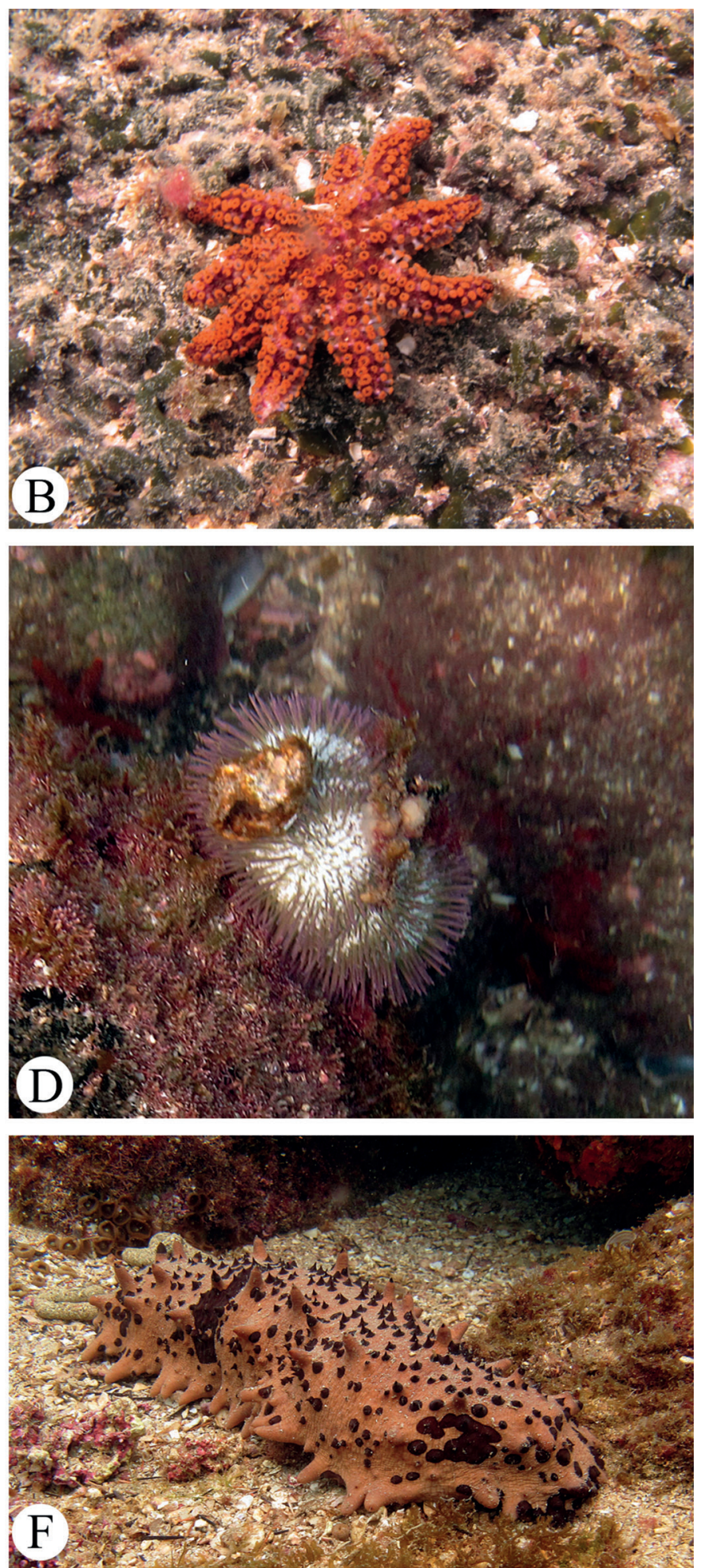

Figure 3. Some echinoderms species from Santa Catarina. (A) Echinaster (Othilia) brasiliensis (Xavier Island; Photo: Nataly Slivak), (B) Coscinasterias tenuispina (Xavier Island; Photo: Nataly Slivak), (C) Mellita quinquiesperforata (Saco do Capim - Arvoredo Island; Photo: Jonathan Lawley), (D) Lytechinus variegatus (Xavier Island; Photo: Nataly Slivak), (E) Ophiothela mirabilis (Photo: Jonathan Lawley), (F) Isosticopus badionotus (Rancho Norte-Arvoredo Island; Photo: Nataly Slivak). 
is already marketed as a delicacy and there is evidence of illegal exploitation of the holothuroids Isostichopus badionotus and Holothuria (H.) grisea in Brazil (Souto \& Martins, 2017). Besides that, echinoderms, such as those recorded in the North and South Bays of Santa Catarina directly associated with the bottom, can be susceptible to contaminants stored in marine sediment caused by the progressive occupation and environmental degradation in favor of urban expansion (Moreira, 2011).

Therefore, studies on reproductive biology, population dynamics and genetics are needed. To achieve this knowledge, it is essential to protect these species. Currently, the Institute of the Environment of Santa Catarina (Instituto do Meio Ambiente de Santa Catarina, IMA), works with environmental protection programs in Hydrographic Basins and Federal Marine-Coastal Conservation Units, such as the Arvoredo Biological Marine Reserve (Reserva Biológica Marinha do Arvoredo), which shelter most echinoderm species listed in this study (IMA, 2011).

\section{CONCLUSION}

Considering the great length of the Brazilian's coastline, information on the occurrence and distribution of extant echinoderms species seems to be insufficient. Regional biodiversity inventories are the first step in understanding coastal ecological processes even at ocean scales. The remarkable number of new records, in recent years, of Echinodermata fauna listed in this study for Santa Catarina, reveals the lack of taxonomic studies. Updated checklists, as presented herein, may help monitor anthropic impacts, foster conservation strategies and generate subsidies for future studies of taxonomy, ecology and related fields.

AUTHORS'CONTRIBUTIONS:NS, AL: Conceptualization; NS: Methodology, Software, Data curation, Formal analysis, Writing - original draft, Visualization, Investigation; NS, AL, HR: Writing - review \& editing; AL: Funding acquisition; AL, HR: Supervision. All authors actively participated in the discussion of the results, they reviewed and approved the final version of the paper.

CONFLICTS OF INTEREST: Authors declare there are no conflicts of interest.

FUNDING INFORMATION: The first author is grateful to Coordenação de Aperfeiçoamento de Pessoal de Nível Superior (CAPES) for the scholarship to support this research project (number process: 942117). Field research in Santa Catarina was also funded by Fundação de Amparo à Pesquisa e Inovação do Estado de Santa Catarina (FAPESC - number process: 4302/2010-8).

ACKNOWLEDGMENTS: We would like to thank the Federal University of Rio Grande do Sul (UFRGS) for the opportunity of a master's degree, the Federal University of Santa Catarina (UFSC) for the laboratory structure, and to the Marine Biological Reserve of Arvoredo for the sample authorization. We are grateful to Dr. Norma Würdig for her dedication to supporting the Master's student.

\section{REFERENCES}

Albuquerque, M. da N.; Campos-Creasey, L.S. \& Guille, A. 2001. Two new species of Amphiuridae (Echinodermata, Ophiuroidea) from the southeastern coast of Brazil. Zoosystema, 23(3): 591-604. https:// sciencepress.mnhn.fr/sites/default/files/articles/pdf/z2001n3a13.pdf.

Alitto, R.A. dos S.; Bueno, M. de L.; Di Domenico, M. \& Borges, M. 2016. Annotated checklist of echinoderms from Araçá Bay, southeastern Brazil. Check List, 12(1): 1-15. https://www.biotaxa.org/c//article/ view/12.1.1836.

Alitto, R.A. dos S.; Granadier, G.; Christensen, A.B.; O'Hara, T.; Di Domenico, M. \& Borges, M. 2020. Unravelling the taxonomic identity of Ophiothela Verrill, 1867 (Ophiuroidea) along the Brazilian coast. Journal of the Marine Biological Association of the United Kingdom, 100(3): 1-14.

Alvarado, J.J. \& Solís-Marín, F.A. 2013. Echinoderm research and diversity in Latin America. Berlin, Spinger Press.

Alves, O.F. de S. \& Cerqueira, W.R.P. 2000. Echinodermata das praias de Salvador (Bahia, Brasil). Revista Brasileira de Zoologia, 17(2): 543-553. https://repositorio.ufba.br/ri/handle/ri/3106.

Alves, R.R.N.; Mota, E.L.S. \& Dias, T.L.P. 2018. Use and commercialization of animals as decoration. In: Alves, R.R.N. \& Albuquerque, U.P. (Eds.). Ethnozoology. Academic Press, 261-275.

Amaral, A.C.Z.; Ribeiro, C.V.; Mansur, M.C.D.; Santos, S.B.; Avelar, W.E.P.; Matthews-Cascon, H.; Leite, F.P.P.; Melo, G.A.S.; Coelho, P.A.; Buckup, G.B.; Buckup, L.; Ventura, C.R.R. \& Tiago, C.G. 2008. Invertebrados aquáticos: a situação de ameaça dos invertebrados aquáticos no Brasil. In: Machado, A.B.M.; Drummond, G.M. \& Paglia, A.P. (Eds.). Livro vermelho da fauna brasileira ameaçada de extinção. vol. 1. Brasília, Fundação Biodiversitas, p. 156-165. https://www.icmbio.gov.br/portal/images/ stories/biodiversidade/fauna-brasileira/livro-vermelho/volumel/Inv aquaticos.pdf.

Anderson, A.B.; Carvalho-Filho, A.; Morais, R.A.; Nunes, L.T.; Quimbayo, J.P. \& Floeter, S.R. 2015. Brazilian tropical fishes in their southern limit of distribution: checklist of Santa Catarina's rocky reef ichthyofauna, remarks and new records. Check List, 11: 1-25. https://www.biotaxa.org/ cl/article/view/11.4.1688.

Barboza, C.A.M.; Mendes, F.M.; Dalben, A. \& Tommasi, L.R. 2010. Echinodermata, Ophiuroidea, Gorgonocephalus Leach, 1815: First report of the genus for the Brazilian continental margin. Check List, 6: 289-291. https://www.biotaxa.org/cl/article/view/6.2.289.

Barrilli, G.H.C. 2018. Avaliação ambiental e estrutura das comunidades de peixes e invertebrados marinhos em áreas de arrasto camaroeiro do litoral de Santa Catarina, Brasil. (PhD thesis). São Carlos, Universidade Federal de São Carlos. http://repositorio.ufscar.br/handle/ufscar/10484.

Birkeland, C. 1989. The influence of echinoderms on coral-reef communities. In: Jangoux, M. \& Lawrence, J.M. (Eds.). Echinoderm Studies. Rotterdan, A.A. Balkema. v. 3, 79p.

Borges, M. 2006. Taxonomia, distribuição e biologia reprodutiva de Ophiuroidea (Echinodermata) das Regiões Sudeste e Sul do Brasil. (PhD thesis). Universidade Estadual Paulista, São Paulo. https://repositorio.unesp.br/ handle/11449/106575.

Borges, M. \& Amaral, A.C.Z. 2007. Ophiuroidea (Echinodermata): quatro novas ocorrências para 0 Brasil. Revista Brasileira de Zoologia, 24(4): 855-864. https://www.scielo.br/scielo. php?pid=S0101-81752007000400001\&script=sci_arttext\&tlng=pt. 
Borges, M. \& Campos, L.D.S. 2011. Ophiomusium acuferum (Ophiolepididae) and Ophiomisidium pulchellum (Ophiuridae) (Ophiuroidea: Echinodermata), redescription based on the Brazilian specimens. Zoologia (Curitiba), 28(2): 219-225. https://www.scielo.br/scielo. php?pid $=$ S1984-46702011000200010\&script $=$ sci arttext.

Borges, M.; Alitto, R.A. do S. \& Amaral, A.C.Z. 2015. From baby to adult: ontogenetic series of nine species of Ophiuroidea from Atlantic Southwestern. Revista de Biología Tropical, 63: 361-381. https://www. sciel0.sa.cr/pdf/rbt/v63s2/0034-7744-rbt-63-s2-361.pdf.

Borges, M.; Monteiro A.M.G. \& Amaral, A.C.Z. 2006. A new species of Ophiomisidium (Echinodermata: Ophiuroidea) from the continental shelf and slope off southern Brazil. Journal of the Marine Biological Association of the United Kingdom, 86(6): 1449-1454.

Borges, M.; Monteiro, A.M.G. \& Amaral, A.C. 2002. Taxonomy of Ophiuroidea (Echinodermata) from the continental shelf and slope of the southern and southern Brazilian coast. Biota Neotropica, 2: 1-69. https://www. scielo.br/pdf/bn/v2n2/a10v2n2.pdf.

Borges, M.; Monteiro, A.M.G. \& Amaral, A.C.Z. 2004. Classe Ophiuroidea. In: Amaral, A.C.Z. \& Rossi-Wongtschowski, C.L. Del B. (Eds.). Série RevizEEScore Sul: Biodiversidade bentonica da região sudeste-sul do BrasilPlataforma Externa e Talude superior. Vol. 1. Instituto Oceanográfico, USP, São Paulo, 156-160. https://www.marinha.mil.br/secirm/sites/www. marinha.mil.br.secirm/files/documentos/revizee/score-sul-6.pdf.

Branco, J.0.; Freitas-Jr., F. \& Christoffersen, M.L. 2015. Bycatch fauna of seabob shrimp trawl fisheries from Santa Catarina state, southern Brazil. Biota Neotropica, 15: 1-14. https://www.scielo.br/pdf/bn/v15n2/16760611-bne-1676-0611-2014-0143.pdf.

Branco, J.0.; Lunardon-Branco, M.J.; Peret, A.C.; Souto, F.X.; Schveitzer, R. \& Vale, W.G. 1998. Associações entre macroinvertebrados e peixes demersais na armação do Itapocoroy, Penha, SC, Brasil. Brazilian Archives of Biology and Technology, 41(2): 1-10. https://www.scielo.br/pdf/babt/ v41n2/v41n2a15.pdf.

Branco, J.0.; Machado, I.F. \& Bovendorp, M.S. 2004. Avifauna associada a ambientes de influência marítima no litoral de Santa Catarina, Brasil. Zoologia, 21(3): 459-466. https://www.scielo.br/pdf/rbzool/ v21n3/21894.pdf.

Briggs, J.C. \& Bowen, B.W. 2012. A realignment of marine biogeographic provinces with particular reference to fish distributions. Journal of Biogeography, 39(1): 12-30.

Brustolin, M.C. 2013. Influência de Encope emarginata (Leske, 1778) (Echinodermata) sobre a estrutura das associações meiobêntica $e$ microfitobêntica de fundos sublitorais estuarinos. (Master thesis). Universidade Federal do Paraná, Curitiba. https://acervodigital.ufpr.br/ handle/1884/30642.

Bueno, M.L. 2015. Biodiversidade dos Echinodermata na porção sul do embaiamento sul brasileiro. (Master thesis). Universidade Estadual de Campinas, Campinas. http://repositorio.unicamp.br/sspui/handle/ REPOSIP/315313.

Bueno, M.L.; Alitto, R.A.S.; Guilherme, P.D.B.; Di Domenico, M. \& Borges, M. 2018. Guia ilustrado dos Echinodermata da porção sul do Embaiamento Sul Brasileiro. Pesquisa e Ensino em Ciências Exatas e da Natureza, 2(2): 169-237. https://dialnet.unirioja.es/servlet/ articulo? codigo $=6903366$.

Calil, P.; Rocha, R.M.; Freire, C.A. \& Roper, J.J. 2009. The role of Asterina stellifera (Echinodermata: Asteroidea) as a predator in a rocky intertidal community in southern Brazil. Zoologia, 26(2): 279-287. https://www. scielo.br/pdf/zool/v26n2/a10v26n2.pdf.

Capel, K.C.C.; Segal, B.; Lindner, A. \& Bertuol, P. 2012. Corallith beds at the edge of the tropical South Atlantic. Coral Reefs, 31: 75. http://doi. org/10.1007/s00338-011-0818-3.
Caregnato, F.F.; Wiggers, F.; Tarasconi, J.C. \& Veitenheimer-Mendes, I.L. 2009. Taxonomic composition of mollusks collected from the stomach content of Astropecten brasiliensis (Echinodermata: Asteroidea) in Santa Catarina, Brazil. Revista Brasileira de Biociências, 7(3): 252-259. http://www.ufrgs. br/seerbio/ojs/index.php/rbb/article/view/1182/869.

Carraro, J.L.F. 2012. Esponjas marinhas do sul do Brasil: estrutura das assembleias, interações e biodiversidade. (PhD thesis). Universidade Federal do Rio Grande do Sul, Rio Grande do Sul. https://www.lume. ufrgs.br/handle/10183/69715.

Carvalho, J.L.B.; Schettini, C.A.F. \& Ribas, T.M. 1998. Estrutura termohalina do litoral centro-norte catarinense. Notas Técnicas da FACIMAR, 2: 181-197. https://siaiap32.univali.br/seer/index.php/bjast/article/ view/2447/1669.

Castellano, G.C. 2009. Manutenção de gradientes de concentração entre o fluido celômico e a água do mar em equinodermos. (Undergraduate thesis). Universidade Federal do Paraná, Curitiba. https://acervodigital.ufpr.br/ handle/1884/30156.

Castellano, G.C.; Lopes, E.M.; Ventura, C.R.R. \& Freire, C.A. 2017. Early time course of variation in coelomic fluid ionic concentrations in sea urchins abruptly exposed to hypo-and hyper-osmotic salinity challenges: Role of size and cross-section area of test holes. Journal of Experimental Zoology Part A: Ecological and Integrative Physiology, 327(9): 542-550.

Cim, R.V. 1999. Distribuição temporal e hábitos alimentares de Asterina stellifera Möbius, 1859 (Echinodermata) em Itapema do Norte, SC. (Undergraduate thesis). Universidade Federal do Paraná, Curitiba. https://www.acervodigital.ufpr.br/handle/1884/32656.

Clark, A. \& Tortonese, E. 1986. Echinaster sepositus madseni n. subsp., from West Africa (Echinodermata: Asteroidea). Atti della Societa Italiana di Scienze Naturali e del Museo Civico di Storia Naturale, Milano, 127: 65-71. https://www.biodiversitylibrary.org/item/267048\#page/149/ mode/1up.

Cunha, C.M.; Padula, V.; Magalhães, A.R.M.; Ferreira, F.M. \& Ferreira, J.F. 2016. Moluscos. In: Lindner, A. (Ed.). Vida Marinha de Santa Catarina. Editora da Universidade Federal de Santa Catarina, Florianópolis, 39-51. http:// biodiversidade.ufsc.br/ebook/Livro Web.pdf.

Dias, T.L.; Neto, N.A.L. \& Alves, R.R. 2011. Molluscs in the marine curio and souvenir trade in NE Brazil: species composition and implications for their conservation and management. Biodiversity and Conservation, 20(11): 2393-2405.

Fagundes, L.M. 2016. Abundância de ouriços-do-mar em costões rochosos rasos de Santa Catarina. (Undergraduate thesis). Universidade Federal de Santa Catarina, Florianópolis.

Fergunson, J. 1969. Feeding activity in Echinaster and its induction with dissolved nutrients. Biological Bulletin, 136(3): 374-384.

Floeter, S.R. \& Soares-Gomes, A. 1999. Biogeographic and species richness patterns of Gastropoda on the southwestern Atlantic. Revista Brasileira de Biologia, 59(4): 567-575. https://www.scielo.br/pdf/rbbio/v59n4/ v59n4a05.pdf.

Floeter, S.R.; Rocha, L.A.; Roberteson, D.R.; Joyeux, J.C.; Smith-Vaniz, W.F.; Wirtz, P.; Edwards, A.J.; Barreiros, J.P.; Ferreira, C.E.L.; Gasparini, J.L.; Brito, A.; Bowen, B.W. \& Bernardi, G. 2008. Atlantic reef fish biogeography and evolution. Journal of Biogeography, 35: 22-47.

Fontanella, F.M. \& Hopkins, T.S. 2003. Preliminary phylogeny of Echinaster (Othilia) from the Gulf of Mexico based on morphological characters (Echinodermata: Asteroidea). In: Féral, J.P. \& David, B. (Eds.). European Conference on Echinoderm Research, 60, 2001. Proceedings. Lisse, A.A. Balkema. p. 91-95.

Freire, C.A.; Santos, I.A. \& Vidolin, D. 2011. Osmolality and ions of the perivisceral coelomic fluid of the intertidal sea urchin Echinometra lucunter (Echinodermata: Echinoidea) upon salinity and ionic challenges. 
Zoologia, 28(4): 479-487. https://www.scielo.br/pdf/zool/v28n4/ v28n4a09.pdf.

Gherardi, D.F.M.; Cabral, A.P.; Klein, A.H.F.; Muehe, D.C.E.H.; Noernberg, M.A.; Tessler, M.G. \& Sartor, S.M. 2008. Mapeamento da sensibilidade ambiental ao óleo da Bacia Marítima de Santos. Brazillian Journal of Aquatic Science and Technology, 12: 11-31. https://siaiap32.univali.br/ seer/index.php/bjast/article/view/658/531.

GBIF: Global Biodiversity Information Facility. Species - The Global Biodiversity Information Facility. Available: http://www.gbif.org. Access: 01/03/2021.

Gondim, A.I.; Christoffersen, M.L \& Dias, T.L.P. 2014. Taxonomic guide and historical review of starfishes in northeastern Brazil (Echinodermata, Asteroidea). Zookeys, 449: 1-56. https://www.ncbi.nlm.nih.gov/pmc/ articles/PMC4233396/pdf/zookeys-449-001.pdf.

Gondim, A.l.; Christoffersen, M.L. \& Dias, T.L.P. 2020. Catalogue of sponges, cnidarians, and echinoderms from Brazil based on a historical inventory of the Invertebrate Collection Paulo Young (CIPY). Turkish Journal of Bioscience and Collections, 4(2): 30-63. https://dergipark.org.tr/en/ download/article-file/1294220.

Gondim, A.I.; Dias, T.L.P.; Campos, F.F.; Alonso, C. \& Christoffersen, M.L. 2011. Macrofauna bêntica do Parque Estadual Marinho de Areia Vermelha, Cabedelo, Paraíba, Brasil. Biota Neotropica, 11(2): 75-86. https://www. scielo.br/pdf/bn/v11n2/09.pdf.

Gondim, A.I.; Dias, T.L.P.; Christoffersen, M.L. \& Stöhr, S. 2015. Redescription of Hemieuryale pustulata von Martens, 1867 (Echinodermata, Ophiuroidea) based on Brazilian specimens, with notes on systematics and habitat association. Zootaxa, 3925(3): 341-360. https://www.mapress.com/ zootaxa/2015/f/z03925p360f.pdf.

Gondim, A.I.; Lacouth, P.; Alonso, C. \& Manso, C.L.C. 2008. Echinodermata da Praia do Cabo Branco, João Pessoa, Paraíba, Brasil. Biota Neotropica, 8: 151-159. https://www.scielo.br/pdf/bn/v8n2/a16v8n2.pdf.

Gondim, A.I.; Manso, C.L.D.C. \& Alonso, C. 2010. First record of Ophionereis dolabriformis (Echinodermata: Ophiuroidea: Ophionereididae) for the Brazilian coast. Marine Biodiversity Records, 3(1): 1-4.

Hadel, V.F.; Monteiro, A.M.G.; Ditadi, A.F.; Tiago, C.G. \& Tommasi, L.R. 1999. Echinodermata. In: Migotto, A.C. \& Tiago, C.G. (Eds.). Biodiversidade do Estado de São Paulo, Brasil: síntese do conhecimento ao final do século XX - Invertebrados Marinhos. São Paulo, Fundação de Amparo à Pesquisa do Estado de São Paulo. v. 3, p. 260-271.

Hopkins, T.S.; Fontanella, F.M. \&Ventura, C.R.R. 2003. Morphological diagnosis of three Brazilian sea stars of the Genus Echinaster (Sub-genus Othilia). In: Féral, J.P. \& David, B. (Eds.). European Conference on Echinoderm Research, 60, 2001. Proceedings. Lisse, A.A. Balkema. p. 97-103.

Hyman, L.H. 1955. The Invertebrates: Echinodermata. New York, McGraw-Hill. v. 4

Instituto Chico Mendes de Conservação da Biodiversidade (ICMBio). 2016. Sumário executivo do Livro Vermelho da Fauna Ameaçada de Extinção. Brasília, Instituto Chico Mendes de Conservação da Biodiversidade. https://www.icmbio.gov.br/ran/images/stories/Noticias/sumario executivo livro vermelho ed 2016.pdf.

Instituto Chico Mendes de Conservação da Biodiversidade (ICMBio). 2018. Livro Vermelho da Fauna Brasileira Ameaçada de Extinção: Invertebrados. v. 7. Brasília, Instituto Chico Mendes de Conservação da Biodiversidade. https://www.icmbio.gov.br/portal/images/stories/comunicacao/ publicacoes/publicacoes-diversas/livro_vermelho_2018_vol7.pdf.

Instituto do Meio Ambiente de Santa Catarina (IMA). 2011. Lista das Espécies da Fauna Ameaçada de Extinção em Santa Catarina. Resolução Consema № 002,06 de dezembro de 2011. Secretaria de Estado do Desenvolvimento Econômico Sustentável (SDS), Florianópolis. https://www.ima.sc.gov.br/ index.php/downloads/biodiversidade/fauna/2432-relatorio-tecnicofinal-lista-de-especies-ameacadas-de-extincao-em-sc.
International Union for Conservation of Nature and Natural Resources (IUCN). 2020. The IUCN Red List Categories and Criteria. Available: https://www. iucnredlist.org. Access: 22/03/2020.

Klein, J.A. 1998. A macrofauna bêntica associada aos bancos da vieira Pecten ziczac no litoral sul do Brasil. (Undergraduate thesis). Universidade Federal do Paraná, Curitiba. https://acervodigital.ufpr.br/handle/1884/33358.

Klein, J.A.; Borzone, C.A. \& Pezzuto, P.R. 2001. A macro e megafauna bêntica associada aos bancos da vieira Euvola ziczac (Mollusca: Bivalvia) no litoral sul do Brasil. Atlântica, 23: 17-26.

Labbé-Bellas, R. 2013. Assembleias de ouriços-do-mar e relações com o habitat em diferentes recifes brasileiros. (Master thesis). Universidade Federal de Santa Catarina, Florianópolis. https://repositorio.ufsc.br/bitstream/ handle/123456789/107576/319156.pdf?sequence=1\&isAllowed=y.

Labbé-Bellas, R.; Cordeiro, C.A.; Floeter, S.R. \& Segal, B. 2016. Sea urchin abundance and habitat relationships in different Brazilian reef types. Regional Studies in Marine Science, 8: 33-40.

Lawley, J.W.; Fonseca, A.C.; Júnior, E.F. \& Lindner, A. 2018. Occurrence of the non-indigenous brittle star Ophiothela of. mirabilis Verrill, 1867 (Echinodermata, 0phiuroidea) in natural and anthropogenic habitats off Santa Catarina, Brazil. Check List, 14: 453. https://checklist.pensoft.net/ article/22591/download/pdf.

Leite-Castro, L.V.; de Souza-Jr., J.; Salmito-Vanderley, C.S.B.; Nunes, J.F.; Hamel, J.F. \& Mercier, A. 2016. Reproductive biology of the sea cucumber Holothuria grisea in Brazil: importance of social and environmental factors in breeding coordination. Marine Biology, 163(67): 1-13.

Lessios, H.A. 1988. Population dynamics of Diadema antillarum (Echinodermata: Echinoidea) following mass mortality in Panama. Marine Biology, 99(4): 515-526. https://www.academia.edu/ download/30813107/Lessios MarBiol 1988.pdf.

Lima, E.J. de B. \& Fernandes, M.L.B. 2009. Diversidade de equinodermos (Echinodermata) no Estado de Pernambuco (Brasil). Revista Brasileira de Zoociências, 11(1): 55-63. http://periodicos.ufjf.br/index.php/ zoociencias/article/view/24321/13583.

Lima, E.J.; Gomes, P.B.\& Souza, J.R. 2009. Reproductive biology of Echinometra lucunter (Echinodermata: Echinoidea) in a northeast Brazilian sandstone reef. Anais da Academia Brasileira de Ciências, 81(1): 51-59. https:// www.scielo.br/pdf/aabc/v81n1/a07v81n1.pdf.

Lindner, A.; Oliveira, A.F.S. de; Batista, A.A.; Freire, A.S.; Ramos, B. de; Giraldes, B.W.; Faria-Jr., E.; Bastos, E. de 0.; Peres, L.M.C.; Fontoura, L.; Sissini, M.N.; Horta, P.A.; Floeter, S.R.; Macedo, T.P.; Almeida, T.C.M. de \& Segal, B. 2017. A biodiversidade marinha das ilhas da REBio Arvoredo e entorno. In: Segal, B.; Freire, A.S.; Lindner, A.; Krajewski, J.P. \& Soldatelli, M. MAArE: Monitoramento Ambiental da Reserva Biológica Marinha do Arvoredo e entorno. Florianópolis, Editora da Universidade Federal de Santa Catarina. p. 201-249.

Lopes, E.M.; Ferreira, C.E.L. \& Ventura, C.R.R. 2017. Occurrence of the genus Pseudoboletia (Echinoidea: Toxopneustidae) in the South-Western Atlantic: morphological and molecular approaches. Revista de Biología Tropical, 65(1): 299-308. https://revistas.ucr.ac.cr/index.php/rbt/article/ view/31698/31375.

Lopes, E.M.; Pérez-Portela, R.; Paiva, P.C. \& Ventura, C.R.R. 2016. The molecular phylogeny of the sea star Echinaster (Asteroidea: Echinasteridae) provides insights for genus taxonomy. Invertebrate Biology, 135(3): 235-244.

Ludwig, H. 1882. Verzeichruiss der Von Prof Dr Ed van Beneden an der Küste von Brasilien Gesammelten Echinodermen. Mémoires Couronnés et Mémoires des Savants Étrangers, 44: 1-26.

Lunardon-Branco, M.J.; Branco, J.0.; Verani, J.R. 2006. Relações tróficas entre macroinvertebrados e peixes, na enseada da Armação do Itapocoroy, Penha, SC. In: Branco, J.0. \& Marenzi, A. (Orgs.). Bases ecológicas para um desenvolvimento sustentável: estudos de caso em Penha, SC. Itajaí, Editora 
da UNIVALI. p. 183-196. http://www.avesmarinhas.com.br/bases\%20 ecologicas-cap\%C3\%ADtul0\%2013.pdf.

MacCord, F.S. \& Duarte, L.F.L. 2002. Dispersion in populations of Tropiometra carinata (Crinoidea: Comatulida) in the Sao Sebastiao Channel, Sao Paulo State, Brazil. Estuarine, Coastal and Shelf Science, 54(2): 219-225.

Magalhães, W.F.; Martins, L.R. \& Alves, O.F.S. 2005. Inventário dos Echinodermata do Estado da Bahia. Brazilian Journal of Aquatic Science and Technology, 9(1): 61-65. http://rediberoamericanaequinodermos. com/wp-content/uploads/2016/02/489.pdf.

Mah, C.L. \& Blake, D.B. 2012. Global diversity and phylogeny of the Asteroidea (Echinodermata). PloS One, 7(4): 1-22. https://journals.plos.org/ plosone/article?id=10.1371/journal.pone.0035644.

Manso, C.L.C. 2004. Echinodermata da Bacia de Camamu, Bahia, Brasil. Biologia Geral experimental, 5(1): 19-25.

Manso, C.L.D.C.; Gondim, A.I. \& Ventura, C.R.R. 2014. New records of Ophiuroidea (Echinodermata) of the Brazilian coast, with notes on its taxonomy and distribution. Marine Biodiversity Records, 7: 1-7.

Marcgrave, J. 1942. História Natural do Brasil. São Paulo, Imprensa Oficial do Estado. [edição facsimili]

Marchiori, M.0.; Kohlmann, C. \& Fontoura, N.F. 1996. Densidade populacional e distribuição de Echinoidea e Asteroidea da Illha do Arvoredo, Santa Catarina, Brasil. In: Congresso Brasileiro de Zoologia, 21ํ. Resumos. Porto Alegre, Sociedade Brasileira de Zoologia, Universidade Federal do Rio Grande do Sul. p. 138.

Mariante, F.L.; Lemos, G.B.; Eutrópio, F.J.; Castro, R.R. \& Gomes, L.C. 2010. Reproductive biology in the starfish Echinaster (Othilia) guyanensis (Echinodermata: Asteroidea) in southeastern Brazil. Zoologia, 27(6): 897-901. https://www.scielo.br/pdf/zool/v27n6/10.pdf.

Martins, L.; Souto, C. \& Menegola, C. 2012a. First record of Holothuria (Theelothuria) princeps and Thyone pawsoni (Echinodermata: Holothuroidea) in the South Atlantic Ocean. Marine Biodiversity Records, 5:1-6.

Martins, L.; Souto, C. \& Menegola, C. 2012b. A new genus and new species of Sclerodactylidae (Holothuroidea: Dendrochirotida) from the southwestern Atlantic coast. Zootaxa, 3506: 54-62.

Martins, L.; Souto, C.; Magalhães, W.F.; Alves, O.F. de S.; Rosa, I.L. \& Sampaio, C.L.S. 2012c. Echinoderm harvesting in Todos-0s-Santos Bay, Bahia State, Brazil: the aquarium trade.SITIENTIBUS série Ciências Biológicas, 12(1):53-59. http:// periodicos.uefs.br/ojs/index.php/sitientibusBiologia/article/view/123/187.

Martins, M.0. \& Almeida, T.C.M. 2014. Distribuição Espacial da Macrofauna e Sua Interação Com o Sedimento no Parque Aquícola da Enseada da Armação do Itapocoroy/SC. Brazilian Journal of Aquatic Science and Technology, 18: 45-59. https://siaiap32.univali.br/seer/index.php/bjast/ article/view/4087/3223.

Martins, L.; Souto, C.; Braga, J. \& Tavares, M. 2018. Echinoidea and Holothuroidea (Echinodermata) of the Trindade and Martin Vaz Archipelago, off Brazil, with new records and remarks on taxonomy and species composition. Journal of the Marine Biological Association of the United Kingdom, 98(3): 521-555.

MCZ-IZ: The Database of the Zoological Collections-Invertebrate Zoology. Museum of Comparative Zoology. Harvard University. Available: https:// mczbase.mcz.harvard.edu. Access: 01/03/2021.

Mendes, F.M.; Marenzi, A.W.C. \& Domenico, M.D. 2006. Population patterns and seasonal observations on density and distribution of Holothuria grisea (Holothuroidea: Aspidochirotida) on the Santa Catarina Coast, Brazil. SPCBeche-de-mer Information Bulletin, 23:5-10. https://coastfish. spc.int/News/BDM/23/BDM23.pdf\#page=5.

Metri, R. 2006. Ecologia de um banco de algas calcárias da reserva biológica marinha do arvoredo, SC, Brasil. (PhD thesis). Universidade Federal do Paraná, Curitiba. https://acervodigital.ufpr.br/handle/1884/4719.
Miranda, A.L.S.; Lima, M.L. de F.; Sovierzoski, H.H. \& Correia, M.D. 2012. Inventory of the Echinodermata collection from the Universidade Federal de Alagoas. Biota Neotropica, 12(2): 1-12. https://www.scielo.br/pdf/ bn/v12n2/v12n2a14.pdf.

Monteiro, A.M.G. 1998. Avaliação das espécies de ofiuróides (Echinodermata) conhecidas na costa brasileira. In: Congresso Brasileiro de Zoologia, $22^{\circ}$. Resumos. Recife, Sociedade Brasileira de Zoologia, Universidade Federal de Pernambuco. p. 197.

Moreira, M.C. 2011. Diversidade, ocorrência e distribuição da fauna de invertebrados demersal das baías Norte e Sul de Florianópolis (SC), Brasil. (Master thesis). Universidade Federal de Santa Catarina, Florianópolis. https://repositorio.ufsc.br/bitstream/ handle/123456789/96077/295156.pdf.

Müller, F. 1898. Observações sobre a fauna marinha da costa de Santa Catarina. Revista do Museu Paulista, 3: 31-40.

Muséum national d'Histoire naturelle (MNHN). [2020] Database. Echinoderms Batabase Collection. Muséum National D'Histoire Naturelle. Available: https://science.mnhn.fr/institution/mnhn/collection/ie/item/search. Access: 01/10/2020.

Nascimento, J.L. do \& Campos, I.B. 2011. Atlas da fauna brasileira ameaçada de extinção em unidades de conservação federais. Brasília, Instituto Chico Mendes de Conservação da Biodiversidade (ICMBio). https://www. icmbio.gov.br/portal/images/stories/documentos/Atlas-ICMBio-web. pdf.

National Museum of Natural History (NMNH). [2020] Database. The Department of Invertebrate Zoology (IZ) collections database. Smithsonian National Museum of Natural History. Available: https://collections. nmnh.si.edu/search/iz. Access: 01/10/2020.

Netto, L.F. 2006. Echinodermata do Canal de São Sebastião, São Sebastião (SP). (Master thesis). Universidade de São Paulo, São Paulo. https://teses. usp.br/teses/disponiveis/41/41133/tde-12092007-152035/publico/ Luiz Fernando Netto.pdf.

Netto, L.F.; Hadel, V.F. \& Tiago, C.G. 2005. Echinodermata from São Sebastião Channel (São Paulo, Brazil). Revista Biología Tropical, 53(3): 207-218. https://revistas.ucr.ac.cr/index.php/rbt/article/view/26777/26960.

OBIS: Ocean Biodiversity Information System. Global biodiversity indices from the Ocean Biogeographic Information System. Intergovernmental Oceanographic Commission of UNESCO. Available: https://obis.org. Access: 01/03/2021.

O'Hara, T.D.; Hugall, A.F.; Cisternas, P.A.; Boissin, E.; Bribiesca-Contreras, G.; Sellanes, J.; Paulay, G.; Byrne, M. 2018a. Phylogenomics, life history and morphological evolution of ophiocomid brittlestars. Molecular Phylogenetics and Evolution, 130: 67-80.

0'Hara, T.D.; Stöhr, S.; Hugall, A.F.; Thuy, B. \& Martynov, A.V. 2018b. Morphological diagnoses of higher taxa in Ophiuroidea (Echinodermata) in support of a new classification. European Journal of Taxonomy, 416: $1-35$.

Oliveira, I.L. 1989. Stelleroidea (Echinodermata) do litoral catarinense. (Undergraduate thesis). Universidade Federal de Santa Catarina, Florianópolis.

Oliveira, I.L. 1990. Equinodermos do litoral catarinense: II. Alguns Stelleroides (Echinodermata) da Ilha de Santa Catarina e arredores. In: Congresso Brasileiro de Zoologia, $1^{\circ}$. Resumos. Londrina, Sociedade Brasileira de Zoologia.

Oliveira, I.L.; Moreira, A.P.T. \& Saalfeld, K. 1987. Levantamento de equinodermes da Ilha de Santa Catarina (Florianópolis, SC): dados preliminares. In: Seminários sobre Ciências do Mar, $2^{\circ}$. Anais. Florianópolis, Universidade Federal de Santa Catarina. p. 18-24.

Oliveira, J.P.; Oliveira, J. de \& Manso, C.L.C. 2010. Inventário da coleção de equinodermos do LABIMAR, Campus Prof. Alberto Carvalho, Universidade 
Federal de Sergipe. Scientia Plena, 6(12): 1-14. https://scientiaplena. emnuvens.com.br/sp/article/view/314/83.

Padula, V.; Bahia, J.; Vargas, C. \& Lindner, A. 2011. Mollusca, Nudibranchia: new records and southward range extensions in Santa Catarina, Southern Brazil. Check List, 7: 806-808. https://www.lindnerlab.ccb.ufsc. br/pdf/Padula_etal_2011.pdf.

Paine, R.T. 1966. Food web complexity and species diversity. The American Naturalist, 100: 65-75. http://www.csun.edu/ msteele/classes/ marine_ecology/readings/Paine_1966.pdf.

Pangestuti, R. \& Arifin, Z. 2017. Medicinal and health benefit effects of functional sea cucumbers. Journal of Traditional and Complemnetary Medicine, 1-11. https://www.sciencedirect.com/science/article/pii/ $\underline{S 222541101730069 X}$.

Queiroz, V.; Sales, L.; Neves, E. \& Johnsson, R. 2013. Holotúrias do litoral da Bahia (Echinodermata: Holothuroidea): Inventário e novos registros. Arquivos de Ciências do Mar, 46(2): 107-112. http://repositorio.ufc.br/ bitstream/riufc/28992/1/2013 art vqueiroz.pdf.

Rathbun, R. 1879. A list of the Brazilian echinoderms, with notes on their distribution, etc. Transactions of the Connecticut Academy of Arts and Sciences, 5: 18-158.

Reichholf, V.J. 1981. Some data on a sample of the sand-dollar Encope emarginata (Leske, 1778) from the Coast of Santa Catarina, Brazil. Spixiana, 4: 111-114.

Riffel, R.F. 2016. Influência de fatores físicos na composição da comunidade bêntica de substrato consolidado em diferentes escalas no litoral de Santa Catarina. (Master thesis). Universidade Federal de Santa Catarina, Florianópolis. https://repositorio.ufsc.br/handle/123456789/167657.

Rodrigues, M.L.G.; Franco, D. \& Sugahara, S. 2004. Climatologia de frentes frias no litoral de Santa Catarina. Revista Brasileira de Geofisica, 22: 135-151. https://www.scielo.br/pdf/rbg/v22n2/a04v22n2.pdf.

Santana, A.; Manso, C.L.; Almeida, A. \& Alves, 0.F. 2020. Taxonomic review of Ophiothrix Müller \& Troschel, 1840 (Echinodermata: Ophiuroidea) from Brazil, with the description of four new species. Zootaxa, 4808(1): 51-78. https://www.mapress.com/j/zt/article/view/zootaxa.4808.1.3.

Santos, C.P.; Coutinho, A.B. \& Hajdu, E. 2002. Spongivory by Eucidaris tribuloides from Salvador, Bahia (Echinodermata: Echinoidea). Marine Biological Association of the United Kingdom. Journal of the Marine Biological Association of the United Kingdom, 82(2): 295.

Segal, B.; Freire, A.S.; Lindner, A.; Krajewski, J.P. \& Soldateli, M. 2017. Monitoramento Ambiental da Reserva Biológica Marinha do Arvoredo e Entorno. Florianópolis, Editora da Universidade Federal de Santa Catarina.

Soares, J.B. \& Resgalla-Jr., C. 2016. Echinodermata in ecotoxicological tests: maintenance and sensitivity. Brazilian Journal of Oceanography, 64: 29-36. https://www.scielo.br/pdf/bjoce/v64n1/1679-8759bjoce-64-01-0029.pdf.

Souto, C. \& Martins, L. 2017. Os Equinodermos. In: Nunes, J.M. de C. \& Matos, M.R.B. de (Eds.). Litoral Norte da Bahia: caracterização ambiental, biodiversidade e conservação. Salvador, EDUFBA. p. 303-315. https:// repositorio.ufba.br/ri/bitstream/ri/24288/3/litornal-norte-RI.pdf.

SpeciesLink. Rede SpeciesLink. Centro de Referência em Informação Ambiental (CRIA), Fundação de Amparo à Pesquisa do Estado de São Paulo (FAPESP). Available: http://www.splink.org.br. Access: 01/03/2021.

Stöhr, S.; Conand, C. \& Boissin, E. 2008. Brittle stars (Echinodermata: Ophiuroidea) from La Réunion and the systematic position of Ophiocanops Koehler, 1922. Zoological Journal of the Linnean Society, 153(3): 545-560. https://academic.oup.com/zoolinnean/article/153/3/ 545/2606424?login=true.

Stöhr, S.; O'Hara, T.D. \& Thuy, B. 2012. Global diversity of brittle stars (Echinodermata: Ophiuroidea). PloS One, 7(3): 1-14. https://journals. plos.org/plosone/article?id=10.1371/journal.pone.0031940.
Tavares, Y.A.G. 1996. Ecologia populacional de Mellita quinquiesperforata (Echinoidea: Clypeasteroidea) em diferentes parias do litoral do Paraná, Brasil. (Master thesis). Universidade Federal do Paraná, Curitiba. http:// repositorio.ufc.br/bitstream/riufc/17674/1/2014 tese iccmdias.pdf.

Tavares, Y.A.G. \& Borzone, C.A. 2015. The reproductive cycle of the sea urchin Lytechinus variegatus (Echinodermata: Echinoidea) in southern Brazil. Revista de Biología Tropical, 63(2): 243-250. https://www.scielo.sa.cr/ $\mathrm{pdf} / \mathrm{rbt} / \mathrm{v} 63 \mathrm{~s} 2 / 0034-7744-\mathrm{rbt}-63-\mathrm{s} 2-243 . \mathrm{pdf}$.

Tiago, C.G. 1998. Dendrochirotida (Echinodermata: Holothuroidea) no Canal de São Sebastião. (PhD thesis). Universidade de São Paulo, São Paulo.

Tiago, C.G. \& Ditadi, A.S.F. 2001. Holothurians from the Brazilian coast: a historical survey. In: Barker, M. (Ed.). International Echinoderm Conference, $10^{\circ}$. Proceedings. New Zealand, Swets \& Zeitlinger Publishers. p. 379-384.

Tommasi, L.R. 1958. El genero Astropyga Gray, nuevo para America del Sud. Neotropica, 4: 85-87.

Tommasi, L.R. 1964. Equinodermes (menos Holothuroidea) recogidos por el barco Emília del IOUSP, en el litoral de Santa Catarina. Neotropica, 10: 112-113.

Tommasi, L.R. 1965. Alguns Amphiuridae (Ophiuroidea) do litoral de São Paulo e Santa Catarina. Contribuições Avulsas do Instituto Oceanográfico da Universidade de São Paulo, Série Oceanografia Biológica, 8: 8-9.

Tommasi, L.R. 1966. Lista dos Equinóides Recentes do Brasil. Contribuições Avulsas do Instituto Oceanográfico da Universidade de São Paulo Série Oceanografia Biológica, 11: 1-50.

Tommasi, L.R. 1970a. Os ofiuróides recentes do Brasil e de regiões vizinhas. Contribuições Avulsas do Instituto Oceanográfico da Universidade de São Paulo Série Oceanografia Biológica, 20: 1-146.

Tommasi, L.R. 1970b. Listas dos asteróides recentes do Brasil. Contribuições Avulsas do Instituto Oceanográfico da Universidade de São Paulo Série Oceanografia Biológica, 18: 1-61.

Tommasi, L.R. 1971. Equinodermes do Brasil. I. Sobre algumas novas espécies e outras pouco conhecidas, para 0 Brasil. Boletim do Instituto Oceanográfico, 20: 1-21.

Tommasi, L.R. 1999. Invertebrados marinhos registrados no litoral brasileiro: Echinodermata recentes e fósseis. São Paulo, 102p.

Tommasi, L.R. 2004. Classes Asteroidea, Crinoides, Holothuroidea e Echinoidea. In: Amaral, A.C.Z. \& Rossi-Wongtschowski, C.L. Del B. (Eds.). Série RevizEE-Score Sul: Biodiversidade bentonica da região sudeste-sul do Brasil-Plataforma Externa e Talude superior, 161-163. https://www. icmbio.gov.br/portal/images/stories/biodiversidade/fauna-brasileira/ livro-vermelho/volumel/Inv_aquaticos.pdf.

Ventura, C.R.R.; Borges, M.; Campos, L.S.; Costa-Lotufo, L.V.; Freire, C.A.; Hadel, V.F.; Manso, C.L.C.; Silva, J.R.M.C.; Tavares, Y. \& Tiago, C.G. 2013. Echinoderm from Brazil: Historical research and the current state of biodiversity knowledge. In: Alvarado, J.J. \& Solís-Marín, F.A. (Eds.). Echinoderm research and diversity in Latin America. Berlin, Spinger Press. p. 301-344.

Ventura, C.R.R.; Lima, R.P.N.; Nobre, C.C.; Veríssimo, I. \& Zama, P.C. 2006. Filo Echinodermata. In: Lavrado, H.P. \& Ignacio, B.L. (Eds.). Biodiversidade bentônica da região central da Zona Econômica Exclusiva brasileira. Rio de Janeiro, Museu Nacional. p. 339-389. https://www.marinha.mil.br/ secirm/sites/www.marinha.mil.br.secirm/files/documentos/revizee/ score-central-vol18.pdf.

Ventura, C.R.R.; Slivak, N.N. \& Labbé-Bellas, R. 2016. Equinodermos. In: Lindner, A. (Ed.). Vida Marinha de Santa Catarina. Florianópolis, Editora da Universidade Federal de Santa Catarina. p. 63-65. http://biodiversidade. ufsc.br/ebook/Livro Web.pdf.

Verrill, A.E. 1868. Notes on Radiata in the Museum of Yale College, with descriptions of new genera and species. N. 4 Notice of the corals and 
Echinoderms collected by Prof. C.F. Hartt, at the Abrolhos Reefs, Province of Bahia, Brazil, 1867. Transactions of the Connecticut Acadademy of Arts and Sciences, 1(2): 351-613. https://www.biodiversitylibrary.org/ page/ 13380325 .

Vidolin, D.; Santos-Gouvea, I.A. \& Freire, C.A. 2002. Osmotic stability of the coelomic fluids of a sea-cucumber (Holothuria grisea) and a starfish (Asterina stellifera) (Echinodermata) exposed to the air during low tide: a field study. Acta Biológica Paranaense, 31(1, 2, 3, 4): 113-121. https:// revistas.ufpr.br/acta/article/view/611/503.

Vieira, D.C.; Noldin, J.A.; Deschamps, F.C. \& Resgalla-Jr., C. 2016. Ecological risk analysis of pesticides used on irrigated rice crops in southern Brazil. Chemosphere, 162: 48-54.

WoRMS:WorldRegister ofMarineSpecies. Available:http://www.marinespecies. org at VLIZ. Access: 01/03/2021. https://doi.org/10.14284/170.
Xavier, L.A.R. 2010. Inventário dos equinodermos do estado de Santa Catarina, Brasil. Brazilian Journal of Aquatic Science Technology, 14: 73-78. https:// siaiap32.univali.br/seer/index.php/bjast/article/view/1358/1825.

Yoshimura, C.Y. 1997. Estudo da distribuição vertical e variação sazonal de espécies da zona entremarés em costão rochoso da praia de Itapema do norte, Santa Catarina, Brasil. (Undergraduate thesis). Universidade Federal do Paraná, Curitiba. https://acervodigital.ufpr.br/handle/1884/37533.

Zacarías-Soto, M.; Olvera-Novoa, M.A.; Pensamiento-Villarauz, S. \& SánchezTapia, I. 2013. Spawning and larval development of the four-sided sea cucumber, Isostichopus badionotus (Selenka, 1867), under controlled conditions. Journal of the World Aquaculture Society, 44(5): 694-705. https://doi.org/10.1111/jwas.12061. 


\section{APPENDICES}

Appendix 1: Scientific bibliographic references used to compile the distribution data of echinoderm species on the coast of Santa Catarina. In the middle column, the type of material found and if it is available online.

\begin{tabular}{|c|c|c|c|}
\hline References & Type & Availability & Species number \\
\hline Lindner et al., 2017 & book chapter & printed & 7 \\
\hline Ventura et al., 2016 & book chapter & online & 18 \\
\hline Marchiori et al., 1996 & conference proceedings & printed & 7 \\
\hline Monteiro, 1998 & conference proceedings & printed & 1 \\
\hline Oliveira et al., 1987 & conference proceedings & printed & 16 \\
\hline Oliveira, 1990 & conference proceedings & printed & 21 \\
\hline Amaral et al., 2008 & endangered species lists & online & 9 \\
\hline ICMBio, 2018 & endangered species lists & online & 6 \\
\hline IMA, 2011 & endangered species lists & online & 16 \\
\hline Nascimento \& Campos, 2011 & endangered species lists & online & 10 \\
\hline Bueno, 2015 & master thesis & printed & 25 \\
\hline Labbé-Bellas, 2013 & master thesis & online & 6 \\
\hline Moreira, 2011 & master thesis & online & 7 \\
\hline Netto, 2006 & master thesis & online & 10 \\
\hline Riffel, 2016 & master thesis & online & 3 \\
\hline Barrilli, 2018 & PhD thesis & online & 3 \\
\hline Borges, 2006 & PhD thesis & online & 4 \\
\hline Metri, 2006 & $\mathrm{PhD}$ thesis & online & 8 \\
\hline Borges et al., 2004 & technical report & online & 12 \\
\hline Tommasi, 2004 & technical report & online & 4 \\
\hline Barboza et al., 2010 & scientific manuscripts & online & 1 \\
\hline Borges \& Amaral, 2007 & scientific manuscripts & online & 2 \\
\hline Borges et al., 2002 & scientific manuscripts & online & 11 \\
\hline Borges et al., 2006 & scientific manuscripts & online & 1 \\
\hline Branco et al., 1998 & scientific manuscripts & online & 5 \\
\hline Branco et al., 2015 & scientific manuscripts & online & 11 \\
\hline Bueno et al., 2018 & scientific manuscripts & online & 20 \\
\hline Calil et al., 2009 & scientific manuscripts & online & 1 \\
\hline Caregnato et al., 2009 & scientific manuscripts & online & 1 \\
\hline Castellano et al., 2017 & scientific manuscripts & online & 3 \\
\hline Freire et al., 2011 & scientific manuscripts & online & 1 \\
\hline Gondim et al., 2014 & scientific manuscripts & online & 8 \\
\hline Labbé-Bellas et al., 2016 & scientific manuscripts & online & 8 \\
\hline Lawley et al., 2018 & scientific manuscripts & online & 1 \\
\hline Lopes et al., 2016 & scientific manuscripts & online & 1 \\
\hline Lopes et al., 2017 & scientific manuscripts & online & 1 \\
\hline Lunardon-Branco et al., 2006 & scientific manuscripts & online & 2 \\
\hline Martins \& Almeida, 2014 & scientific manuscripts & online & 2 \\
\hline Mendes et al., 2006 & scientific manuscripts & online & 1 \\
\hline Müller, 1898 & scientific manuscripts & online & 1 \\
\hline Rathbun, 1879 & scientific manuscripts & online & 4 \\
\hline Reichholf, 1981 & scientific manuscripts & online & 1 \\
\hline Santana et al., 2020 & scientific manuscripts & online & 3 \\
\hline Soares \& Resgalla-Jr., 2016 & scientific manuscripts & online & 4 \\
\hline Tommasi, 1964 & scientific manuscripts & printed & 11 \\
\hline Tommasi, 1965 & scientific manuscripts & printed & 1 \\
\hline Tommasi, 1966 & scientific manuscripts & printed & 2 \\
\hline Tommasi, 1970a & scientific manuscripts & printed & 5 \\
\hline Tommasi, 1970b & scientific manuscripts & printed & 2 \\
\hline Vidolin et al., 2002 & scientific manuscripts & online & 2 \\
\hline Vieira et al., 2016 & scientific manuscripts & online & 1 \\
\hline Xavier, 2010 & scientific manuscripts & online & 33 \\
\hline Castellano, 2009 & undergraduate thesis & online & 3 \\
\hline Cim, 1999 & undergraduate thesis & online & 1 \\
\hline Fagundes, 2016 & undergraduate thesis & printed & 4 \\
\hline Klein, 1998 & undergraduate thesis & online & 3 \\
\hline Oliveira, 1989 & undergraduate thesis & printed & 12 \\
\hline Yoshimura, 1997 & undergraduate thesis & online & 1 \\
\hline
\end{tabular}




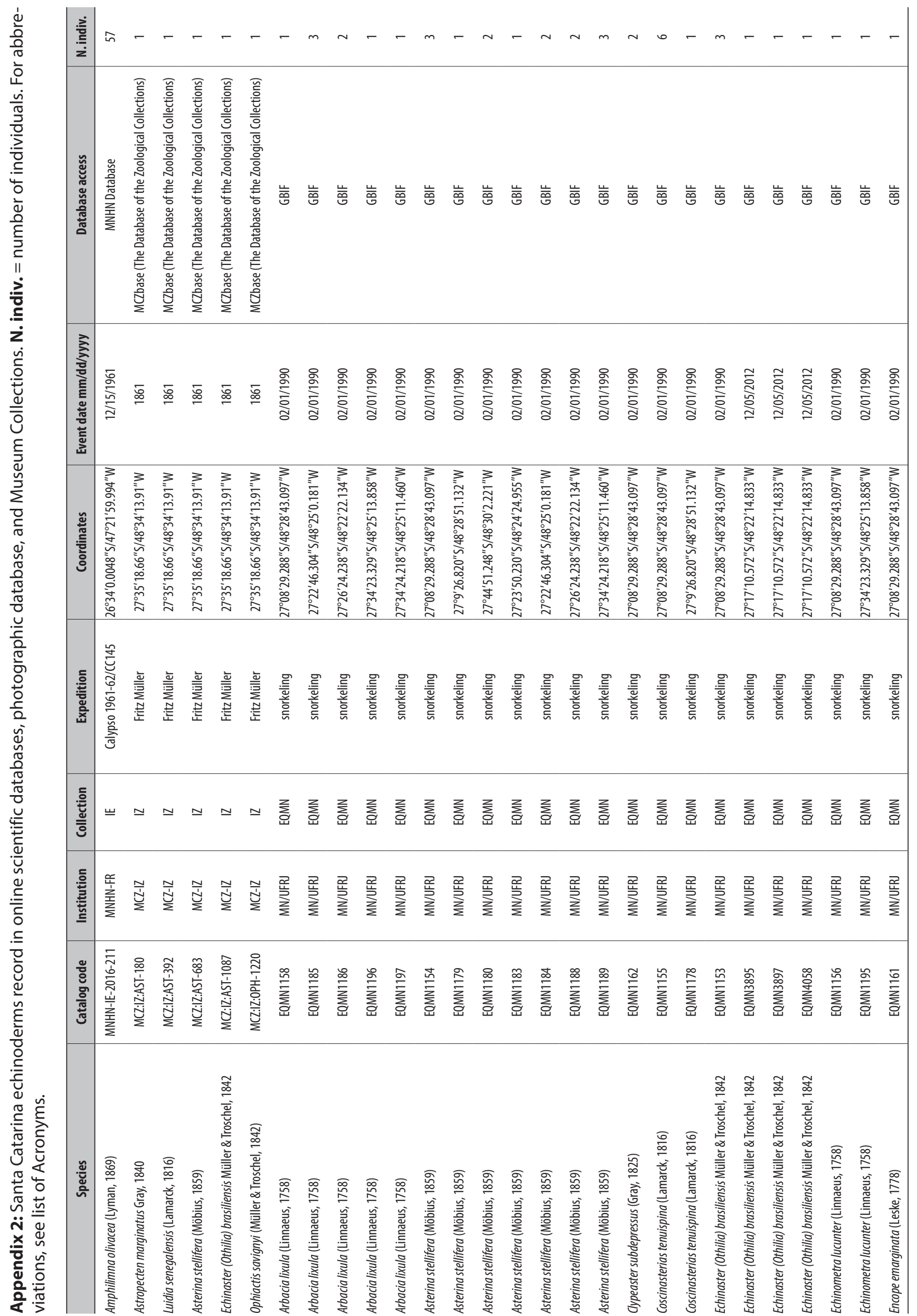




\begin{tabular}{|c|c|c|c|c|c|c|c|c|c|c|c|c|c|c|c|c|c|c|c|c|c|c|c|c|c|c|c|c|}
\hline 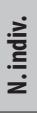 & $\circ$ & - & - & - & $\sim$ & $m$ & - & $\sigma$ & $m$ & - & in & $\infty$ & $\sim$ & $\circ$ & $\sim$ & $\sigma$ & - & $\sim$ & $\sim$ & $\sim$ & \pm & - & - & n & 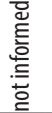 & - & - & - \\
\hline 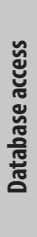 & 和 & 步 & 嵜 & 嵜 & $\frac{1}{\overline{0}}$ & 皚 & 寅 & 嵩 & 崽 & 荘 & 嵜 & 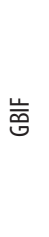 & 嵃 & 崽 & 䱏 & 珤 & $\sum_{\sum}^{\overline{1}}$ & 空 & 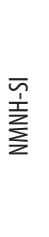 & $\sum_{\substack{\text { 至 } \\
\sum}}$ & 空 & $\sum_{\sum}^{\overline{1}}$ & $\begin{array}{l}\text { 耎 } \\
\sum_{\Sigma}\end{array}$ & $\sum_{\sum}^{\overline{1}}$ & 苂 & 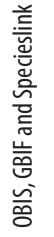 & 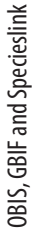 & 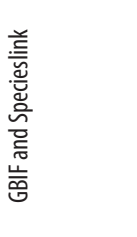 \\
\hline 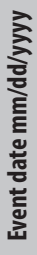 & $\frac{\text { S }}{\stackrel{\sigma}{\sigma}}$ & $\frac{\text { S. }}{\stackrel{\delta}{\sigma}}$ & $\frac{\text { 응 }}{\stackrel{\delta}{\delta}}$ & $\frac{\text { 응 }}{\stackrel{\delta}{\sigma}}$ & $\frac{\text { ㅇ }}{\stackrel{2}{\sigma}}$ & 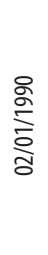 & 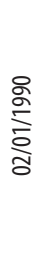 & $\frac{\text { S }}{\stackrel{0}{\vdots}}$ & $\frac{\text { o }}{\stackrel{\sigma}{\sigma}}$ & 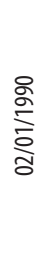 & 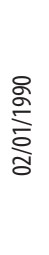 & 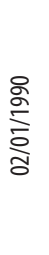 & 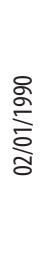 & $\frac{\stackrel{8}{\circ}}{\stackrel{\delta}{\sigma}}$ & 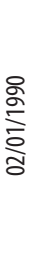 & 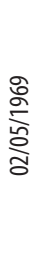 & $\frac{a}{\sigma}$ & 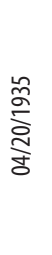 & $\frac{\frac{0}{\sigma}}{\frac{5}{\sigma}}$ & 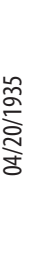 & 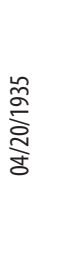 & 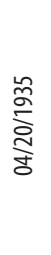 & 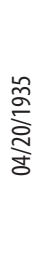 & $\frac{n}{\tilde{\sigma}}$ & $\sum_{\delta}^{\stackrel{\infty}{\vdots}}$ & 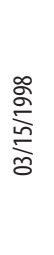 & $\frac{\stackrel{\infty}{\sigma}}{\frac{o}{\sigma}}$ & 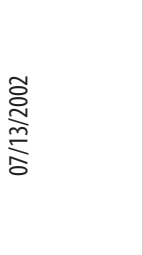 \\
\hline 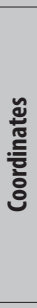 & 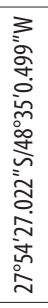 & 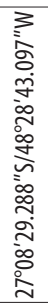 & 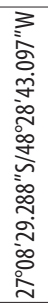 & 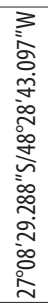 & 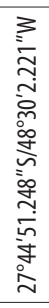 & 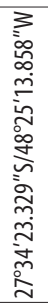 & 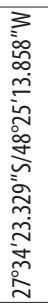 & 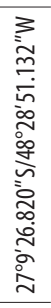 & 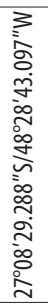 & 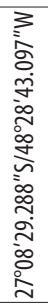 & 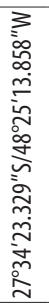 & 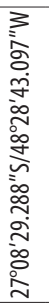 & 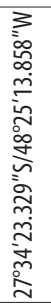 & 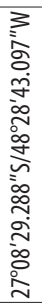 & 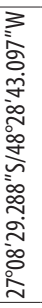 & 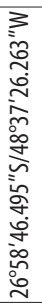 & 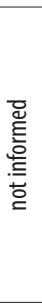 & 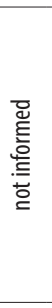 & 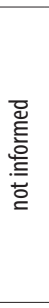 & 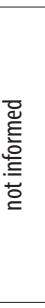 & 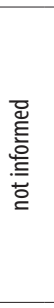 & 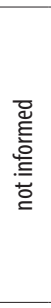 & 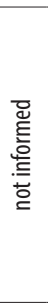 & 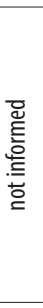 & 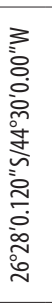 & 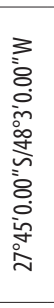 & 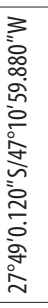 & 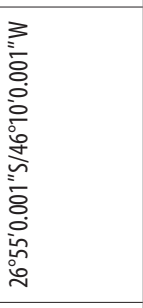 \\
\hline 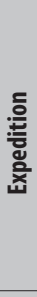 & $\begin{array}{l}\text { 营 } \\
\text { 产 } \\
\text { 咅 }\end{array}$ & 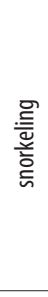 & 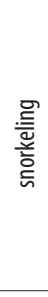 & 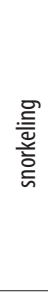 & 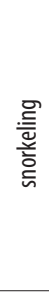 & 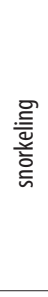 & $\begin{array}{l}\frac{9}{\underline{\underline{E}}} \\
\text { 产 } \\
\text { 号 }\end{array}$ & 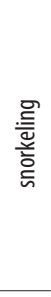 & 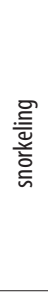 & 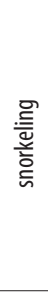 & 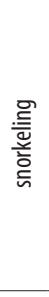 & 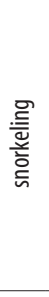 & 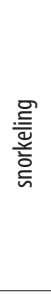 & 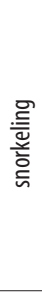 & 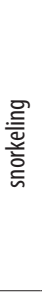 & 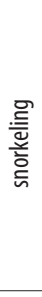 & 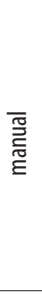 & 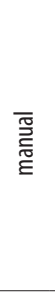 & 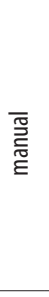 & 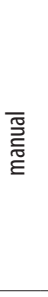 & $\begin{array}{l}\text { 丞 } \\
\text { 吾 }\end{array}$ & 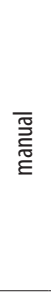 & 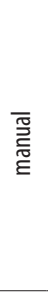 & 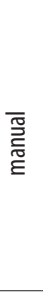 & 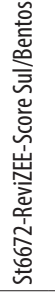 & 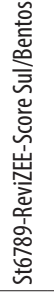 & 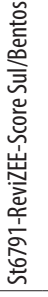 & 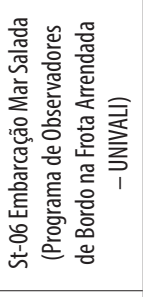 \\
\hline $\begin{array}{l}\frac{\overline{0}}{\underline{\underline{U}}} \\
\overline{\bar{\omega}}\end{array}$ & $\sum_{\text {d }}^{z}$ & 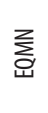 & 竞 & 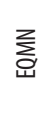 & 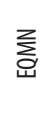 & $\sum_{\text {d }}^{z}$ & $\sum_{\text {d }}^{\text {D }}$ & 竞 & 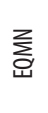 & 竞 & 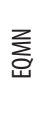 & $\sum_{\text {总 }}$ & $\sum_{\text {d }}^{z}$ & $\sum_{\text {d }}^{2}$ & 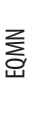 & 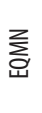 & $\simeq$ & $\simeq$ & $\simeq$ & $\simeq$ & 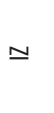 & $\simeq$ & $\simeq$ & $\simeq$ & $\begin{array}{l}\text { 퐇 } \\
\text { 열 }\end{array}$ & $\begin{array}{l}\text { 퐁 } \\
\text { 岁 }\end{array}$ & $\begin{array}{l}\text { ⿳ㅗㅇ } \\
\text { 岁 }\end{array}$ & $\begin{array}{l}\text { 퐁 } \\
\text { 쏠 }\end{array}$ \\
\hline 克 & $\stackrel{\text { 总 }}{\sum_{\Sigma}}$ & $\stackrel{\text { 吝 }}{\sum}$ & $\stackrel{\text { 吝 }}{\sum_{i}}$ & 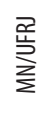 & $\stackrel{\text { 总 }}{\sum_{\Sigma}}$ & $\stackrel{\text { 总 }}{\sum_{\Sigma}}$ & $\sum_{\sum}^{\stackrel{P}{\mid}}$ & 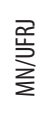 & $\stackrel{\text { 㞼 }}{\sum}$ & 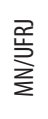 & $\sum_{\sum}^{\stackrel{2}{\mathbf{2}}}$ & 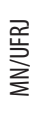 & $\sum_{\sum}^{\text {妾 }}$ & $\sum_{\sum}^{\text {变 }}$ & 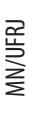 & 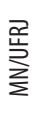 & $\sum_{\sum}^{\overline{1}}$ & 窣 & 窒 & 舍 & 窒 & $\begin{array}{l}\text { 窨 } \\
\sum_{\Sigma}\end{array}$ & $\sum_{\sum_{\Sigma}^{\overline{1}}}^{\overline{\sum_{2}}}$ & $\sum_{\sum}^{\overline{1}}$ & 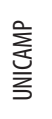 & 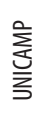 & 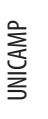 & 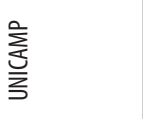 \\
\hline 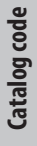 & 总 & 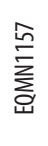 & 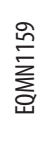 & 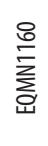 & $\sum_{\sum_{d j}^{\infty}}^{\infty}$ & 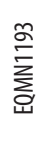 & 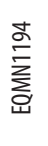 & $\stackrel{\equiv}{\sum_{\text {¿ }}^{E}}$ & 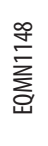 & 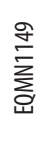 & $\frac{8}{\sum_{0 . j}^{\frac{8}{4}}}$ & 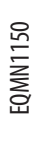 & 离 & 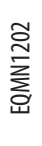 & 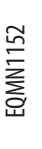 & 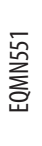 & 忍 & $\begin{array}{l}\text { 总 } \\
\text { 㟋 } \\
\text { 离 }\end{array}$ & 总 & 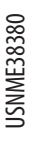 & 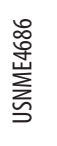 & 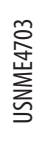 & 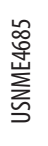 & $\begin{array}{l}\text { 各 } \\
\text { 离 } \\
\text { 峛 }\end{array}$ & 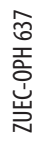 & 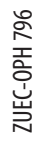 & 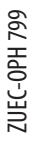 & 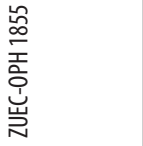 \\
\hline 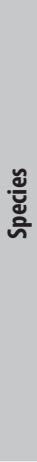 & 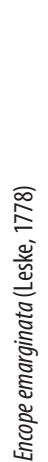 & 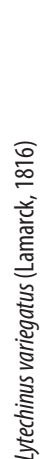 & 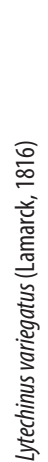 & 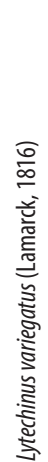 & 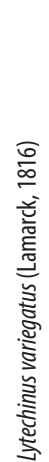 & 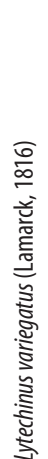 & 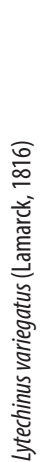 & 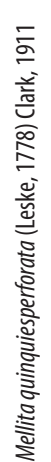 & 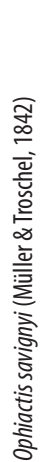 & 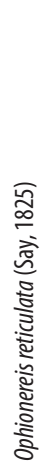 & 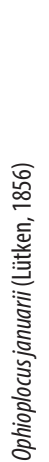 & 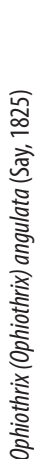 & 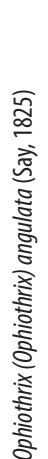 & 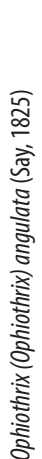 & 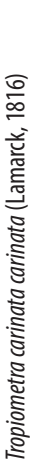 & 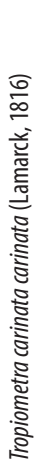 & 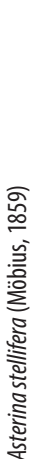 & 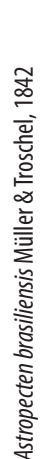 & 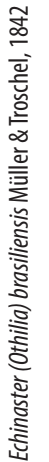 & 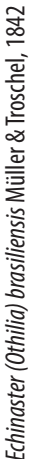 & 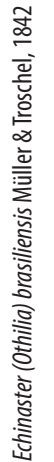 & 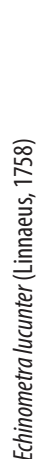 & 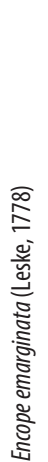 & 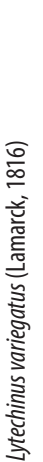 & 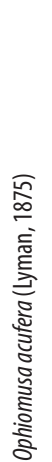 & 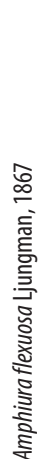 & 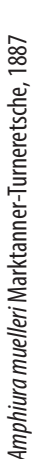 & 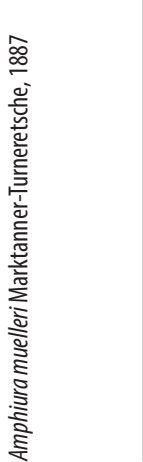 \\
\hline
\end{tabular}




\begin{tabular}{|c|c|c|c|c|c|c|c|c|c|c|c|c|c|c|c|c|c|c|}
\hline 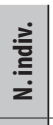 & - & - & - & - & - & - & 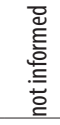 & $\sigma$ & $\sim$ & 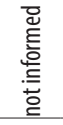 & - & $\stackrel{\circ}{\simeq}$ & - & - & - & $\sim$ & - & - \\
\hline 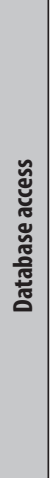 & 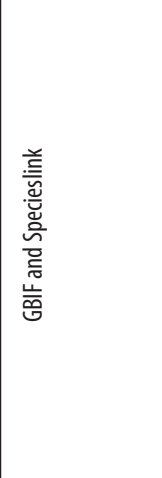 & 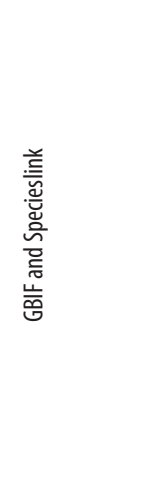 & 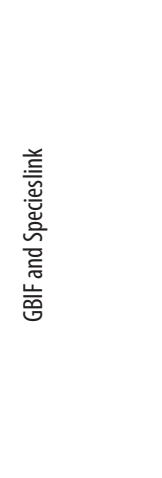 & 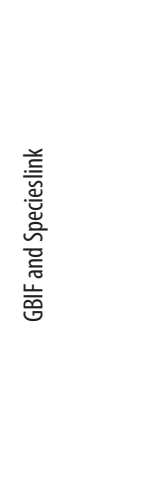 & 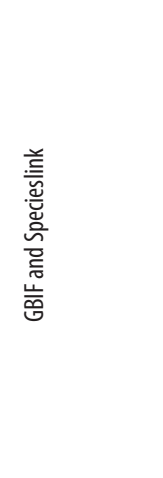 & 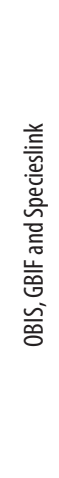 & $\stackrel{\check{0}}{0}$ & 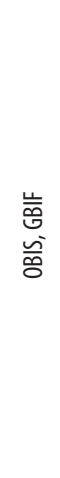 & 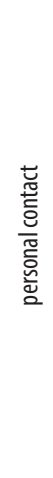 & 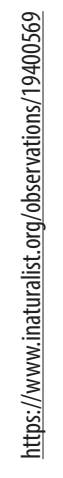 & 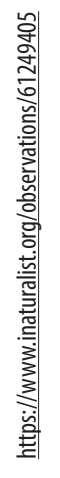 & 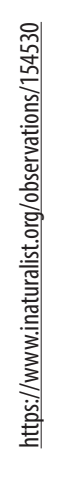 & 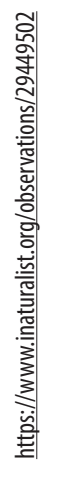 & 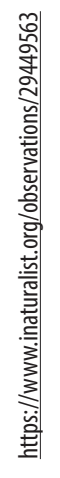 & 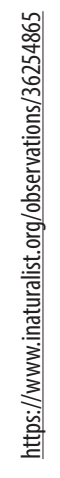 & 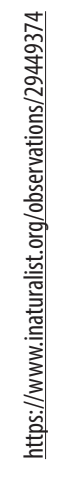 & 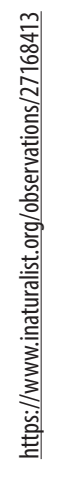 & 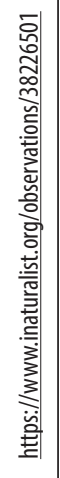 \\
\hline 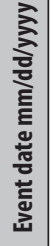 & 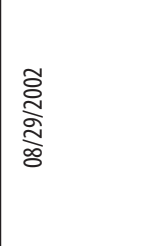 & 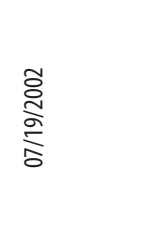 & 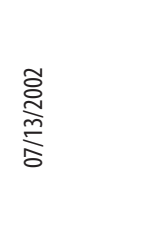 & 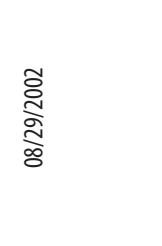 & 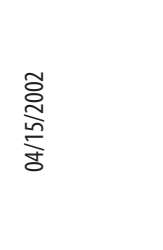 & 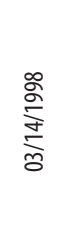 & $\sum_{\vdots}^{\stackrel{\infty}{\vdots}}$ & $\begin{array}{l}\frac{\infty}{\circ} \\
\frac{\delta}{\sigma} \\
\frac{\delta}{\sigma}\end{array}$ & $\begin{array}{l}\text { 品 } \\
\stackrel{\Xi}{ }\end{array}$ & 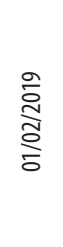 & 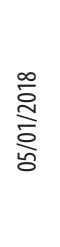 & 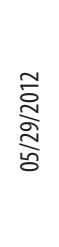 & 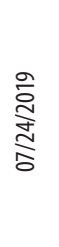 & 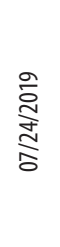 & 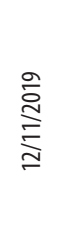 & 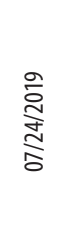 & $\frac{\stackrel{\circ}{\bar{~}}}{\frac{m}{8}}$ & 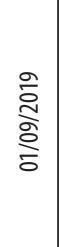 \\
\hline 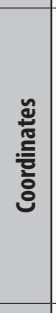 & 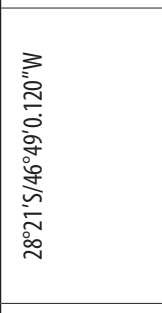 & 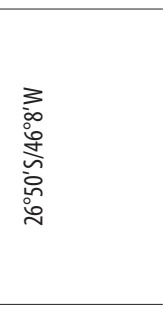 & 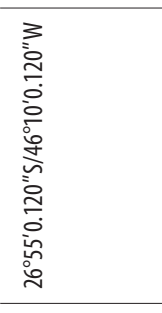 & 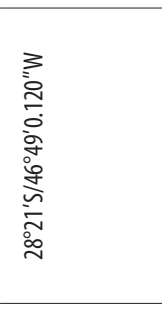 & 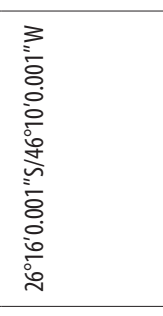 & 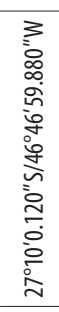 & 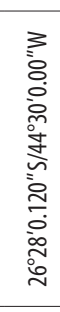 & 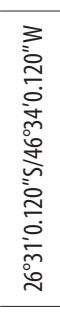 & 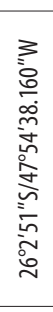 & 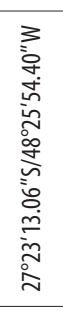 & 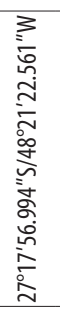 & 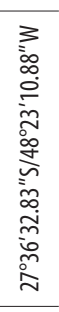 & 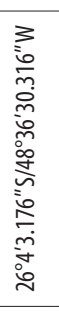 & 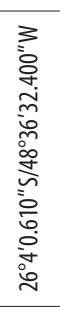 & 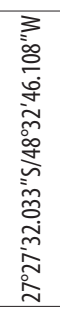 & 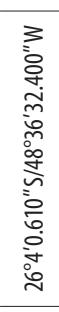 & 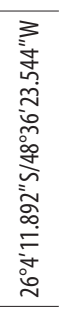 & 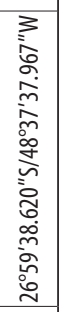 \\
\hline 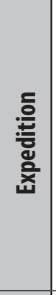 & 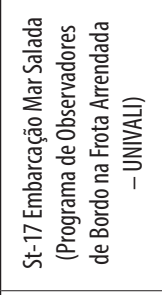 & 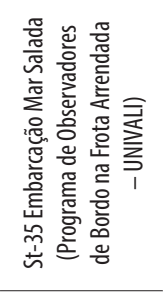 & 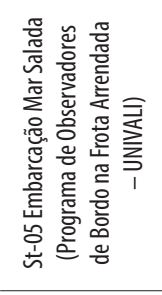 & 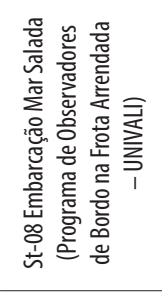 & 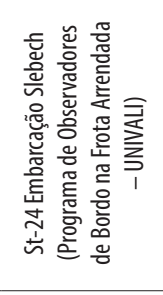 & 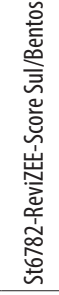 & 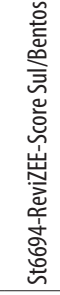 & 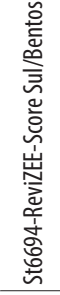 & 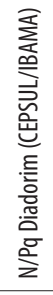 & 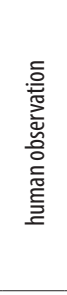 & $\begin{array}{l}\text { 흘 } \\
\text { 离 } \\
\text { 产 } \\
\text { 产 } \\
\text { 产 }\end{array}$ & 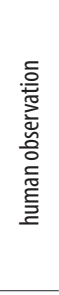 & 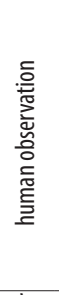 & 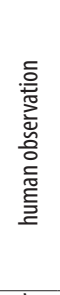 & 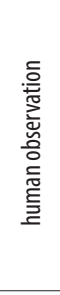 & 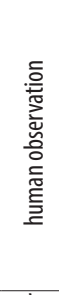 & 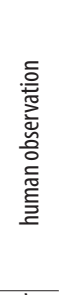 & 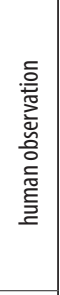 \\
\hline 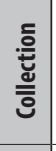 & \begin{tabular}{|l} 
퐁 \\
岁
\end{tabular} & $\begin{array}{l}\text { 퐁 } \\
\text { 岁 }\end{array}$ & $\begin{array}{l}\text { 퐁 } \\
\text { 岁 }\end{array}$ & $\begin{array}{l}\text { 퐁 } \\
\text { 岁 }\end{array}$ & $\begin{array}{l}\text { 퐁 } \\
\text { 岁 }\end{array}$ & $\begin{array}{l}\text { 홍 } \\
\text { 멸 }\end{array}$ & $\begin{array}{l}\text { 홍 } \\
\text { 岁 }\end{array}$ & $\begin{array}{l}\text { 퐁 } \\
\text { 岁 }\end{array}$ & 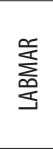 & 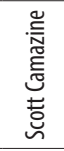 & 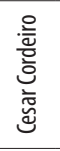 & 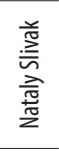 & 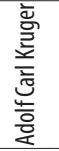 & 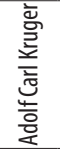 & 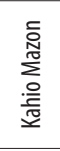 & 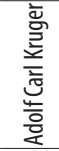 & 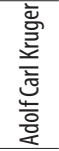 & 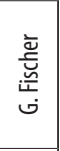 \\
\hline 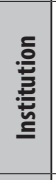 & 妾 & 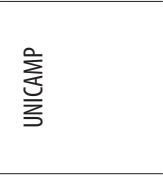 & 妾 & 妾 & $\begin{array}{l}\text { 妾 } \\
\text { 至 }\end{array}$ & 氛 & $\begin{array}{l}\text { 妾 } \\
\overline{3}\end{array}$ & 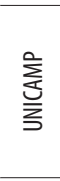 & 育 & 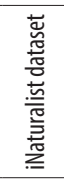 & 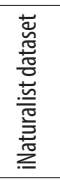 & 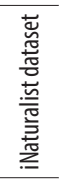 & 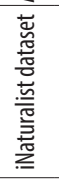 & 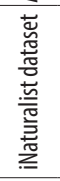 & 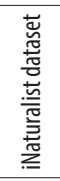 & 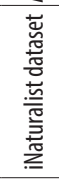 & 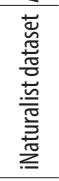 & 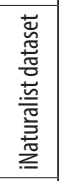 \\
\hline 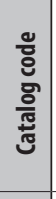 & 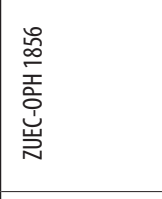 & 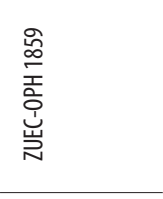 & 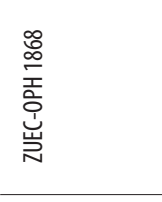 & 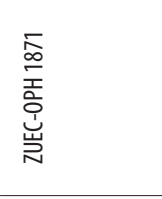 & 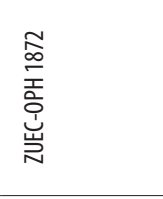 & 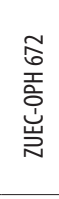 & 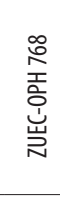 & 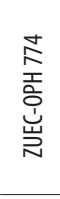 & 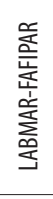 & 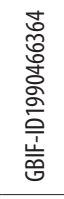 & 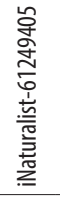 & 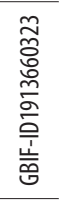 & 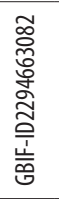 & 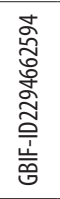 & 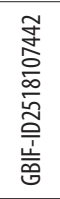 & 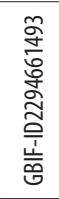 & 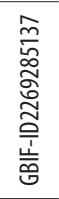 & 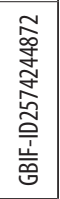 \\
\hline 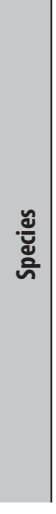 & 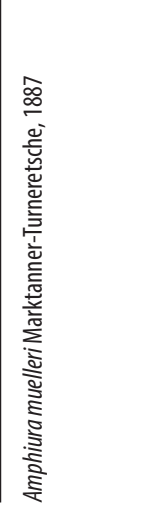 & 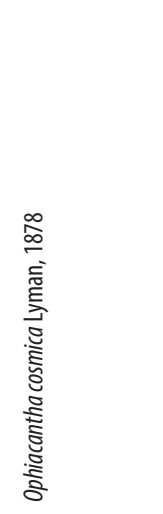 & 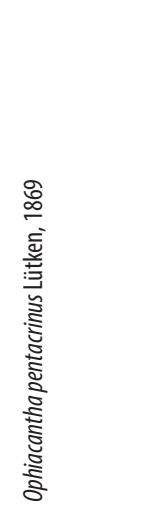 & 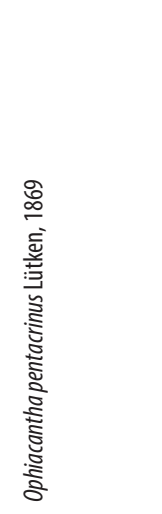 & 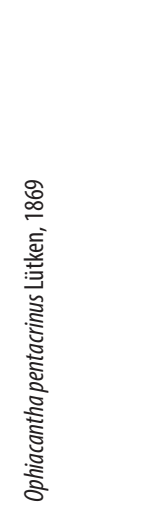 & 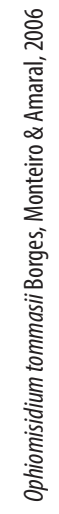 & 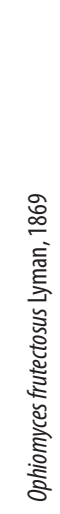 & 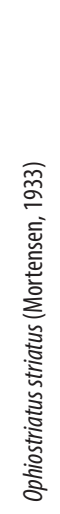 & 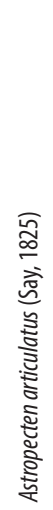 & 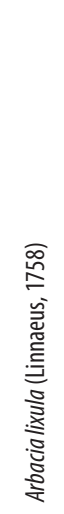 & 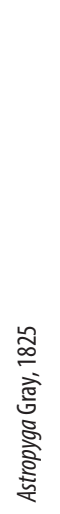 & 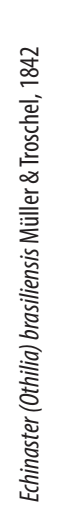 & 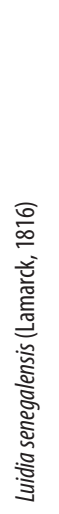 & 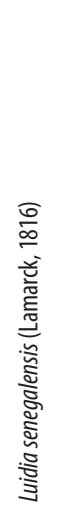 & 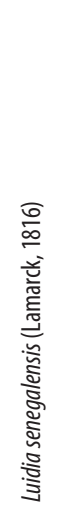 & 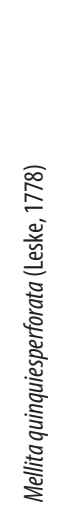 & 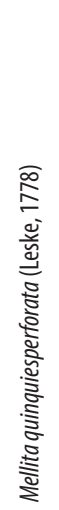 & 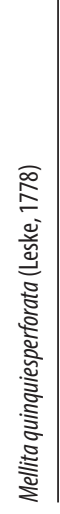 \\
\hline
\end{tabular}

